NBER WORKING PAPER SERIES

\title{
SPATIAL EQUILIBRIA: THE CASE OF TWO REGIONS
}

\author{
Konstantin Kucheryavyy \\ Gary Lyn \\ Andrés Rodríguez-Clare \\ Working Paper 29592 \\ http://www.nber.org/papers/w29592 \\ NATIONAL BUREAU OF ECONOMIC RESEARCH \\ 1050 Massachusetts Avenue \\ Cambridge, MA 02138 \\ December 2021, Revised March 2023
}

We thank Treb Allen, Costas Arkolakis, Dominick Bartelme, Arnaud Costinot, and Cecile Gaubert for very useful conversations and Piyush Panigrahi for excellent research assistance. Konstantin Kucheryavyy acknowledges financial support of JSPS Kakenhi Grants Number 17K13721, 20K13476, and 20H01487 as well as financial support of the HSE University Basic Research Program. An earlier version of the paper titled: "Necessary and Sufficient Conditions for Uniqueness in Spatial Equilibria: The Case of Two Locations," analyzed the case with symmetric trade costs and no agricultural sector. All errors are our own. The views expressed herein are those of the authors and do not necessarily reflect the views of the National Bureau of Economic Research.

NBER working papers are circulated for discussion and comment purposes. They have not been peer-reviewed or been subject to the review by the NBER Board of Directors that accompanies official NBER publications.

(C) 2021 by Konstantin Kucheryavyy, Gary Lyn, and Andrés Rodríguez-Clare. All rights reserved. Short sections of text, not to exceed two paragraphs, may be quoted without explicit permission provided that full credit, including $(\mathcal{C}$ notice, is given to the source. 
Spatial Equilibria: The Case of Two Regions

Konstantin Kucheryavyy, Gary Lyn, and Andrés Rodríguez-Clare

NBER Working Paper No. 29592

December 2021, Revised March 2023

JEL No. F10,F20,R0,R13

\section{$\underline{\text { ABSTRACT }}$}

In this paper we characterize the set of equilibria in a generalized version of the canonical tworegion economic geography model that nests the class of models in Allen and Arkolakis (2014) as well as Krugman (1991) and features an input-output loop. We provide sufficient conditions for uniqueness of equilibria that - in contrast to the well-know result in Allen and Arkolakis (2014) - allow for positive agglomeration externalities, which concentrate economic activity, even in the absence of congestion effects, which disperse it, and highlight the key role played by three additional parameters: the trade elasticity, which regulates the strength of the dispersion force associated with the decline in the terms of trade caused by migration into a region; trade costs, which weaken this dispersion force by limiting trade across regions; and the importance of the agricultural sector, which pushes against agglomeration forces in manufacturing.

Konstantin Kucheryavyy

University of Tokyo

Graduate School of Public Policy

Tokyo 113-0033, Japan

kucher@pp.u-tokyo.ac.jp

Gary Lyn

Department of Economics

Iowa State University

Ames, IA 50011

garyl.lyn@gmail.com
Andrés Rodríguez-Clare

University of California at Berkeley

Department of Economics

Berkeley, CA 94720-3880

and NBER

arc@berkeley.edu 


\section{Introduction}

Recently there has been a surge of research on quantitative models of the spatial distribution of economic activity that are tractable yet sophisticated enough to capture first-order features of the data, such as heterogeneous geography, productivity, and amenities, along with trade and migration across regions. ${ }^{1}$ Given that economies of agglomeration constitute a central feature in economic geography, a challenge in this literature is the possibility of multiple equilibria and its implications for counterfactual analysis.

In their seminal paper, Allen and Arkolakis (2014, henceforth AA) provided a sufficient condition for uniqueness of spatial equilibria for an important class of economic geography models featuring trade and agglomeration forces in a single productive sector with regionally differentiated varieties, as in Armington (1969), and congestion forces, as in Helpman (1998). This sufficient condition hinges on the balance between the elasticity of localized external economies of scale, $\psi \geq 0$, and the elasticity of congestion externalities, $\delta \geq 0$, requiring that the latter be weakly stronger than the former, $\psi \leq \delta$. A benefit of this condition is that, if it holds, it ensures uniqueness regardless of the values of all other parameters, including trade costs. However, it is a strong condition that, for example, rules out agglomeration externalities if there are no congestion externalities. Moreover, this sufficiency result does not tell us anything about the set of equilibria if the condition is violated.

In this paper we characterize the set of equilibria in a generalized version of the canonical two-region economic geography model that nests the class of models in Allen and Arkolakis (2014) as well as Krugman (1991) and features an input-output loop. Section 2 presents the model. There are two sectors, manufacturing and agriculture. Manufacturing is modeled as in Armington (1969), with elasticity of substitution $\sigma>1$ across the goods produced by different regions - so that the trade elasticity is $\varepsilon=\sigma-1>0-$ and features external economies of scale with elasticity $\psi \geq 0$. In addition to labor, manufacturing production employs intermediate goods - a CES aggregator of all regional varieties with elasticity of substitution $\sigma-$ in a Cobb-Douglas fashion with cost share $1-\zeta$. Agriculture consists of a single homogenous good that is subject to frictionless trade. There are two types of labor: manufacturing workers are perfectly mobile across regions and face congestion externalities with elasticity $\delta \geq 0$, while agricultural workers are exogenously located. Preferences are Cobb-Douglas with a share $\beta$ of income spent on manufacturing. The model is isomorphic to Krugman (1991) if $\alpha \equiv \frac{(\psi-\zeta \delta) \varepsilon}{1+\delta}=1$, and

\footnotetext{
${ }^{1}$ See Redding (2022) for a recent survey of the literature.
} 
so we say that $\alpha=1$ corresponds to the "Krugman case". ${ }^{2}$ The AA model obtains when there is no agriculture, $\beta=1$, and AA's sufficient condition for uniqueness corresponds to $\alpha \leq 0$. $^{3}$

Section 3 presents a series of results that characterize the set of equilibria for given values of $\alpha, \varepsilon, \beta, \zeta$, iceberg trade costs, and the distribution of exogenous productivity, amenities, and the number of agricultural workers across the two regions. After showing that irregular equilibria (i.e., equilibria in which all manufacturing workers locate in a single region) are possible only when $\alpha \geq 1$, we show that a regular equilibrium (i.e., an equilibrium in which manufacturing workers are located in both regions) is fully characterized by the relative price of manufacturing goods produced in the two regions (adjusted by the trade elasticity), which we denote by $x$, and that a regular equilibrium satisfies $V(x)=0$ for an explicit function $V(\cdot)$. Thus, characterizing the set of regular equilibria corresponds to characterizing the set of zeros of the function $V(x)$. We show that AA's sufficient condition for uniqueness, $\alpha \leq 0$, continues to hold even in the presence of an input-output loop and an agricultural sector. More importantly, we derive a set of conditions that ensure uniqueness of equilibrium as long as $\alpha \in(0,1)$ and then show how these results are affected when $\alpha=1$, as in the Krugman case. ${ }^{4}$

Broadly speaking, the results of Section 3 establish sufficient conditions for uniqueness of equilibria that - in contrast to the well-know result in Allen and Arkolakis (2014) - allow for positive agglomeration externalities even in the absence of congestion effects, and highlight the key role played by three additional parameters: the trade elasticity, which regulates the strength of the dispersion force associated with the decline in the terms of trade caused by migration into a region; trade costs, which weaken this dispersion force by limiting trade across regions; and the importance of the agricultural sector, which pushes against agglomeration forces in manufacturing.

To shed light on these different forces it proves convenient to consider two special cases: the case with no agriculture, as in AA, and the case with symmetric regions, as in Krugman (1991). Section 4 studies the case with no agriculture, $\beta=1$, and derives a full characterization of the set of equilibria, including necessary and sufficient conditions for uniqueness. These conditions show that if $\alpha \in(0,1)$ then the equilibrium is unique if

\footnotetext{
${ }^{2}$ In Krugman (1991) there is no congestion, $\delta=0$, nor intermediate goods, $\zeta=1$, implying that $\alpha=$ $\psi \varepsilon=1$. Another difference is that in Krugman (1991) economies of agglomeration arise by way of free entry and love of variety rather than through technological external economies of scale, but it is well known that the two approaches lead to identical results - see AA for a details.

${ }^{3}$ Strictly speaking, the AA model has no input-output loops, $\zeta=1$.

${ }^{4}$ We also show that generally the economy can have up to five regular equilibria, and the AA's case with no agriculture can yield at most three regular equilibria.
} 
trade costs are low while there are multiple equilibria if trade costs are high. ${ }^{5}$ Moreover, a lower trade elasticity expands the range of trade costs under which the equilibrium is unique. These results reveal how the terms-of-trade dispersion force can lead to uniqueness even if agglomeration dominates congestion, $\psi>\delta$, so that $\alpha>0$. Intuitively, terms of trade worsen for a region experiencing a rise in population, and this effect is more severe with a lower trade elasticity and when trade costs are low so that there is more trade and terms-of-trade changes have a larger impact on real wages. ${ }^{6}$

The presence of agriculture changes these results in intuitive ways, as shown in Section 5 for the case with symmetric regions. In this case and for $\alpha \in(0,1)$ the number of equilibria depends on the relative importance of manufacturing and agriculture captured by $\beta$ and trade costs for any given value of the trade elasticity. Not surprisingly, the results described above for the case with no agriculture continue to hold when $\beta$ is close to one. At the other extreme, when $\beta$ is low enough then the weight of agriculture is so strong that there is a unique equilibrium for all trade costs. For intermediate values of $\beta$ uniqueness holds if trade costs are either low or high: when both agriculture and manufacturing are important, uniqueness requires trade costs either be low so that terms-of-trade dispersion force is strong enough or high so that dispersion effects associated with the agricultural sector are strong enough. ${ }^{7}$

Finally, the presence of an input-output loop also affects the set of equilibria in intuitive ways. A higher share of intermediates in production of the differentiated manufactured goods, as captured by a lower value of parameter $\zeta$, makes multiplicity more likely through two channels: a lower value of $\zeta$ increases the strength of agglomeration economies and weakens the agricultural sector dispersion force.

We also study whether regular equilibria are stable in the sense that the relative utility in a region is decreasing in the number of manufacturing workers residing in this region

${ }^{5}$ In Section 3 we show that if $\alpha<1$ then the equilibrium is unique if trade costs are low enough even with $\beta<1$. In contrast, the result that there are multiple equilibria for high enough trade costs requires $\beta$ to be high enough.

${ }^{6}$ If there are no congestion externalities on local amenities, $\delta=0$, then $\alpha<1$ is equivalent to $\psi<1 / \varepsilon$. Thus, if the terms-of-trade dispersion force - whose strength is regulated by $1 / \varepsilon-$ dominates agglomeration effects - whose strength is regulated by $\psi$ - then uniqueness is guaranteed under frictionless trade even in the absence of congestion effects. In the context of a multi-sector gravity model of international trade with sector-level external economies of scale, Kucheryavyy, Lyn and Rodríguez-Clare (forthcoming) also show that if the scale elasticity is lower than the inverse of the trade elasticity in every sector then there is a unique equilibrium under frictionless trade.

${ }^{7}$ While the results for the symmetric case suggest that decreasing $\alpha$ or $\beta$ would always make it more likely that the equilibrium is unique, in the Online Appendix we show that this is not always true under asymmetry: depending on other parameters, increasing $\alpha$ or $\beta$ might result in intermittent regions of uniqueness and multiplicity. We also show that while increasing the asymmetry between the two regions tends to enlarge the set of parameters under which the equilibrium is unique, this is also not universally true. 
(the same definition as in the original Krugman, 1991 paper). This analysis is straightforward thanks to the explicit function $V(x)$ : a regular equilibrium is locally stable if and only if $V^{\prime}(x)>0$ evaluated at that equilibrium. We show that for $\alpha \in(0,1)$ if a regular equilibrium is unique then it is locally stable, while for $\alpha>1$ if a regular equilibrium is unique then it is unstable. ${ }^{8}$ For the knife-edge case with $\alpha=1$ a unique regular equilibrium can be stable or unstable depending on other parameter values.

The original Krugman (1991) paper introduced the so-called "no-black-hole condition" as well as the "tomahawk diagram" to describe the patterns of equilibrium labor allocations in manufacturing when the no-black-hole condition holds. We generalize the no-black-hole condition to the case of an input-output loop and show that the tomahawk diagram is qualitatively unaffected by the presence of an input-output loop. We then show that the patterns of equilibria significantly change in the case with $\alpha \in(0,1)$. Specifically, there are four different patterns of labor allocations depending (among other things) on the strength of agglomeration forces. For weak agglomeration forces the equilibrium is unique. For lower to medium strengths of agglomeration forces the pattern of labor allocations is a displaced ellipse. For medium to higher strengths of agglomeration forces the pattern of labor allocations is a displaced tomahawk. And for strong agglomeration forces the pattern of labor allocations is a pitchfork.

The completely symmetric case of the Krugman (1991) model was analyzed in RobertNicoud (2005), who formally showed that the Krugman (1991) model can have at most three regular equilibria: one locally stable and two locally unstable. Sidorov (2011) generalized the results from Robert-Nicoud (2005) by allowing for asymmetries in the distribution of agricultural labor across two regions and providing sufficient conditions for existence and uniqueness of equilibria (along with stability analysis) in an otherwise standard Krugman (1991) model. We generalize even further the results from Sidorov (2011) by allowing for asymmetries in trade costs, productivities, and amenities across regions, adding congestion externalities and an input-output loop. We also allow for $\alpha<1$, thus nesting the AA model. Importantly, we provide a comprehensive characterization of equilibria for a generalized version of the canonical two-region model that integrates all the forces in the AA model as well as those in Krugman (1991). ${ }^{9}$

\footnotetext{
${ }^{8}$ More precisely, for $\alpha \in(0,1)$ if a regular equilibrium is unique then it is generically locally stable in the sense that for all parameter combinations $V^{\prime}(x) \geq 0$, and the set of parameter combinations for which $V^{\prime}(x)=0$ has measure zero.

${ }^{9}$ We provide parsimonious sufficient conditions for existence and uniqueness of equilibria that are much sharper than conditions in Sidorov (2011) even for the cases where we restrict our setup to the one considered in Sidorov (2011).
} 


\section{The Model}

Here we consider a multi-region spatial model as in AA with the inclusion of an inputoutput loop in the differentiated goods sector and an agricultural sector as in Krugman (1991). There are $N$ regions indexed by $i, j$ and $n$, and two sectors: a manufacturing sector with a differentiated good associated with each region, as in the Armington approach, and an agricultural sector producing a homogeneous good. There are two factors of production: labor specific to agriculture and labor specific to manufacturing.

Each region $i$ has $\bar{L}_{i}^{A} \geq 0$ agricultural workers who cannot move across regions and inelastically supply their labor to the agricultural sector. Production technology in agriculture is the same across all regions: the agricultural good is produced one-to-one from agricultural labor. Agricultural goods produced by different regions are perfect substitutes in consumption and can be costlessly traded across regions. Thus, the price of the agricultural good is equal to the wage of agricultural workers and is the same across all regions.

In addition to the agricultural workers, the economy has $\bar{L}$ manufacturing workers who can move freely across regions. Manufacturing workers residing in region $i$ inelastically supply their labor to the manufacturing sector in that region. Production of the manufactured good in region $i$ uses labor and intermediate good according to a CobbDouglas technology,

$$
q_{i}=\zeta^{-\zeta}(1-\zeta)^{-(1-\zeta)} A_{i} L_{i}^{\zeta} Q_{i}^{1-\zeta},
$$

where $q_{i}$ and $L_{i}$ are output of the manufactured good and employment of manufacturing workers in region $i, Q_{i}$ is the amount of the intermediate good produced and used in region $i$, and $\zeta \in(0,1]$. Productivity shifter $A_{i}$ is endogenous and is given by $A_{i} \equiv \bar{A}_{i} L_{i}^{\psi}$, where $\bar{A}_{i}$ is an exogenous component of productivity, and $\psi \geq 0$ regulates the strength of agglomeration externalities affecting manufacturing production. Trade in manufactured goods between regions is subject to iceberg trade costs: delivering a unit of a manufactured good from region $i$ to region $n$ requires shipping $\tau_{n i} \geq 1$ units of the good, with $\tau_{i i}=1$ for all $i$ and $\tau_{n l} \leq \tau_{n i} \tau_{i l}$ for all $n, l$, and $i$ (triangular inequality).

In each region, manufactured goods produced by all regions are aggregated by a constant-elasticity-of-substitution (CES) function with elasticity of substitution $\sigma>1$ to produce a manufactured aggregate, which is then used for consumption and as an intermediate good. Both agricultural and manufacturing workers residing in region $i$ derive utility from a local amenity as well as from consumption of the agricultural good and a manufactured composite. Utility from consumption of the agricultural good and the 
manufactured aggregate is Cobb-Douglas with share $\beta>0$ of expenditure devoted to manufactures. Letting $w_{n}$ and $P_{n}$ denote the wage and price index in manufacturing in region $n$ and $w^{A}$ the wage in agriculture, the welfare of manufacturing workers residing in region $n$ is given by

$$
U_{n}=\frac{w_{n}}{P_{n}^{\beta}\left[w^{A}\right]^{1-\beta}} u_{n}
$$

where $u_{n}$ is the amenity in region $n$ and is given by

$$
u_{n} \equiv \bar{u}_{n} L_{n}^{-\delta}
$$

Here $\bar{u}_{n}$ is an exogenous utility component of amenity in $n$ and $\delta \geq 0$ governs the strength of congestion externalities affecting utility.

All markets are perfectly competitive. Letting $p_{n i}$ denote the price of the manufactured good produced in region $i$ and sold in region $n$, we have $p_{n i}=\tau_{n i} w_{i}^{\zeta} P_{i}^{1-\zeta} / A_{i}$ and

$$
P_{n}=\left[\sum_{j=1}^{N} \bar{A}_{j}^{\varepsilon} L_{j}^{\varepsilon \psi}\left(\tau_{n j} w_{j}^{\zeta} P_{j}^{1-\zeta}\right)^{-\varepsilon}\right]^{-\frac{1}{\varepsilon}},
$$

where $\varepsilon \equiv \sigma-1$ is the trade elasticity. In order to produce the intermediate good, region $n$ buys $\lambda_{n i} P_{n} Q_{n}$ worth of the manufactured good produced in each region $i$, where $\lambda_{n i} \equiv \bar{A}_{i}^{\varepsilon} L_{i}^{\varepsilon \psi}\left(\tau_{n i} w_{i}^{\zeta} P_{i}^{1-\zeta}\right)^{-\varepsilon} P_{n}^{\varepsilon}$. Since $\lambda_{n i}$ is also the share of manufacturing expenditure that region $n$ consumers allocate to imports from $i$, the market clearing condition for the manufactured good in region $i$ is given by

$$
p_{i i} q_{i}=\sum_{n=1}^{N} \lambda_{n i}\left[\beta\left(w_{n} L_{n}+w^{A} \bar{L}_{n}^{A}\right)+P_{n} Q_{n}\right] .
$$

The Cobb-Douglas assumption in (1) implies that $P_{n} Q_{n}=(1-\zeta) p_{n n} q_{n}$ and $w_{n} L_{n}=$ $\zeta p_{n n} q_{n}$, and hence $P_{n} Q_{n}=(1-\zeta) w_{n} L_{n} / \zeta$. Thus, the market clearing condition (5) in region $i$ is

$$
w_{i} L_{i} / \zeta=\sum_{n=1}^{N} \lambda_{n i}\left[\beta\left(w_{n} L_{n}+w^{A} \bar{L}_{n}^{A}\right)+(1-\zeta) w_{n} L_{n} / \zeta\right]
$$

The other equilibrium conditions are that the global market for the agricultural good clears; that welfare of manufacturing workers is equalized across all inhabited regions; and that the global market for manufacturing labor clears. Formally, these conditions are 
given by

$$
\begin{aligned}
& \sum_{i=1}^{N} w^{A} \bar{L}_{i}^{A}=(1-\beta) \sum_{i=1}^{N}\left(w_{i} L_{i}+w^{A} \bar{L}_{i}^{A}\right) \\
& L_{i} \geq 0, \quad \bar{U}-U_{i} \geq 0, \quad L_{i}\left(\bar{U}-U_{i}\right)=0, \quad \text { for all } i \\
& \sum_{i=1}^{N} L_{i}=\bar{L}
\end{aligned}
$$

where $\bar{U}>0$ is the utility level of manufacturing workers. We assume that $\sum_{i=1}^{N} \bar{L}_{i}^{A}>0$ for the versions of the model with $\beta<1$, and that $\sum_{i=1}^{N} \bar{L}_{i}^{A}=0$ for the versions of the model with $\beta=1$ (no agricultural sector).

Following AA, we call an equilibrium regular, if all regions are inhabited, and we call an equilibrium irregular otherwise.

\section{Spatial Equilibria with Two Regions}

In this section we characterize equilibria of the economy described in Section 2 in the general case with two regions $(N=2)$. We first introduce the following definitions: $\gamma \equiv \bar{L}_{1}^{A} /\left(\bar{L}_{1}^{A}+\bar{L}_{2}^{A}\right), \bar{\gamma} \equiv 1-\gamma, \bar{\beta} \equiv 1-\beta, \phi_{1} \equiv \tau_{12}^{-\varepsilon}$ and $\phi_{2} \equiv \tau_{21}^{-\varepsilon}$,

$$
\begin{gathered}
d_{1} \equiv \gamma \zeta \bar{\beta} \phi_{2}^{-1}+(1-\gamma \zeta \bar{\beta}) \phi_{1} \quad \text { and } \quad d_{2} \equiv \bar{\gamma} \zeta \bar{\beta} \phi_{1}^{-1}+(1-\bar{\gamma} \zeta \bar{\beta}) \phi_{2} \\
\chi \equiv \frac{\psi-\zeta \delta}{1+\delta}, \quad \alpha \equiv \chi \varepsilon, \quad \mu \equiv \beta \chi+1-\zeta \bar{\beta}
\end{gathered}
$$

and

$$
G \equiv\left(\bar{u}_{1} / \bar{u}_{2}\right)^{\alpha+\zeta \varepsilon}\left(\bar{A}_{1} / \bar{A}_{2}\right)^{\varepsilon} .
$$

Our restrictions $\psi \geq 0, \delta \geq 0, \beta>0$, and $\zeta \in(0,1]$ imply that $\chi>-\zeta$ and $\mu>1-\zeta$, while parameter $\alpha$ can take any value.

Our analysis in this section reveals that in the case of two regions, the set of equilibria is affected only through parameters $\chi, \alpha, \mu, \phi_{1}, \phi_{2}, d_{1}, d_{2}$, and $G$. This means, for example, that the presence of congestion effects as captured by $\delta \neq 0$ does not have an independent effect on the set of equilibria given $\chi$. Parameters $G$ and $\gamma$ capture asymmetries between the two regions (in Section 5 we consider the case of symmetric regions, which entails $G=1$ and $\gamma=1 / 2)$. As will become clear later, parameter $\alpha$ captures the strength of the net agglomeration force given by $\chi$ relative to the terms-of-trade dispersion force given by $1 / \varepsilon$, while parameter $\mu$ captures the strength of the net agglomeration force relative 
to the dispersion force associated with the agricultural sector (captured by parameter $\beta$ ). Parameters $d_{1}$ and $d_{2}$ capture the interaction of the agricultural-sector dispersion force (adjusted for the presence of an input-output loop and captured by parameter $\zeta \bar{\beta}$ ) with trade costs and the share of agricultural labor in each location, $\gamma$. We will discuss these parameters in more detail as we proceed with the analysis of the model.

Our model nests various different cases that we consider below. What we label the "Krugman case" obtains with $\alpha=1$, while the AA case obtains with $\beta=1$ and $\sum_{i=1}^{N} \bar{L}_{i}^{A}=$ 0 (no agriculture). To see why $\alpha=1$ captures the Krugman model, imagine that $\delta=0$, and note that then $\alpha=1$ entails $\psi=1 / \varepsilon=1 /(\sigma-1)$ : this is the standard result that in a model with monopolistic competition with free entry and CES preferences, there are aggregate economies of scale arising from love of variety with a scale elasticity given by $1 /(\sigma-1)$ (see for example Allen and Arkolakis (2014)). Also, with $\alpha=1$, condition $\mu<1$ is equivalent to $\beta<\frac{\zeta \varepsilon}{\zeta \varepsilon+1}$, which is a generalization of the familiar "no-black-hole condition" from Krugman (1991). We acknowledge that the original Krugman and AA models do not feature input-output loops in manufacturing $(\zeta=1)$, however we show below that introducing an input-output loop does not qualitatively affect the characterization of equilibria in these models in the case of two regions.

We start by characterizing irregular equilibria of the economy of Section 2 with $N=2$.

\section{Proposition 1 (Irregular equilibria).}

(i) If $\alpha<1$ then all equilibria are regular.

(ii) If $\alpha=1$ then:

(ii.a) Allocation $L_{1}=\bar{L}$ and $L_{2}=0$ is an equilibrium if and only if

$$
G \geq \bar{\gamma} \zeta \bar{\beta} \phi_{2}^{\mu-1}+(1-\bar{\gamma} \zeta \bar{\beta}) \phi_{1} \phi_{2}^{\mu} .
$$

(ii.b) Allocation $L_{1}=0$ and $L_{2}=\bar{L}$ is an equilibrium if and only if

$$
G \leq\left[\gamma \zeta \bar{\beta} \phi_{1}^{\mu-1}+(1-\gamma \zeta \bar{\beta}) \phi_{1}^{\mu} \phi_{2}\right]^{-1} .
$$

(iii) If $\alpha>1$ then both patterns of irregular allocations - (1) $L_{1}=\bar{L}$ and $L_{2}=0$, and (2) $L_{1}=0$ and $L_{2}=\bar{L}$ - constitute equilibria.

The proof of Proposition 1 is provided in Appendix B.1. Parts (i) and (iii) of Proposition 1 can easily be extended to more than two regions, and their proofs are very similar to the corresponding proofs in Allen and Arkolakis (2014) (which do not have an agricultural sector nor input-output loops).

The rest of this section is devoted to the analysis of regular equilibria of the two-region economy of Section 2. Namely, we provide conditions under which the economy has a 
unique regular equilibrium and specify whether this equilibrium is stable. ${ }^{10}$ Our definition of stability extends the one in Krugman (1991) to the case of nonzero congestion externalities. We refer to a regular equilibrium as globally stable if the relative utility in region $1, \omega \equiv U_{1} / U_{2}$, is decreasing with the number of manufacturing workers residing in this region, $L_{1}$. When there are multiple equilibria, or when the global stability condition does not hold, we investigate a weaker condition of local stability: we refer to a regular equilibrium as locally stable if the derivative $d \omega / d L_{1}$ evaluated at the equilibrium point is negative. If $d \omega / d L_{1}$ evaluated at the equilibrium point is positive, then we call such an equilibrium unstable. Finally, if $d \omega / d L_{1}$ evaluated at the equilibrium point is zero, then we say that this equilibrium is neither stable nor unstable.

To proceed with the analysis of regular equilibria, we let $x$ denote the price of the manufacturing good produced in region 1 relative to region 2 adjusted by the trade elasticity,

$$
x \equiv\left(\frac{w_{1}^{\zeta} P_{1}^{1-\zeta} /\left(\bar{A}_{1} L_{1}^{\psi}\right)}{w_{2}^{\zeta} P_{2}^{1-\zeta} /\left(\bar{A}_{2} L_{2}^{\psi}\right)}\right)^{\varepsilon} .
$$

In Appendix B.2 we show that the equilibrium system of equations (6)-(9) for regular equilibria can be described in terms of $x$ only. Thus, if we know $x$ we can use equations (6)-(9) to solve for the rest of the equilibrium variables. In turn, $x$ is determined by the equilibrium condition that utilities are equalized across the two regions, $U_{1}=U_{2}$. For the analysis of uniqueness and stability of regular equilibria, it is most convenient to work with this condition in the logarithmic form written as $\ln \omega=0$. In terms of $x$, this can be written as $V(x)=0$, where $V(x)$ is positively proportional to $\ln \omega$ and is defined as

$$
V(x) \equiv \ln G-\alpha \ln \frac{\phi_{1}}{\phi_{2}}+(1-\alpha) \ln x+\alpha \ln g_{d}(x)+\mu \ln g_{\phi}(x)
$$

where $g_{d}(x) \equiv\left(1+d_{1} x\right) /\left(d_{2}+x\right)$ and $g_{\phi}(x) \equiv\left(1+\phi_{1} x\right) /\left(\phi_{2}+x\right) .{ }^{11}$ Functions $g_{d}$ and

\footnotetext{
${ }^{10}$ We emphasize that when we refer to "a unique regular equilibrium", as for example in Propositions 2 and 3 below, it means that there is one regular equilibrium, but there may also be irregular equilibria. To arrive at overall uniqueness results we need to couple these results on regular equilibria with those in Proposition 1.

${ }^{11}$ In the trivial case in which trade is frictionless $\left(\phi_{1}=\phi_{2}=1\right.$, implying also $\left.d_{1}=d_{2}=1\right)$ and there are no agglomeration or congestion externalities $(\psi=0$ and $\delta=0)$, equation $V(x)=0$ boils down to $x^{1 / \varepsilon}=\left(\bar{u}_{2} / \bar{u}_{1}\right)^{\zeta}\left(\bar{A}_{2} / \bar{A}_{1}\right)$, which simply says that the relative price of the manufacturing good has to equal the inverse ratio of productivities adjusted by amenities.
} 
$g_{\phi}$ are related to the model variables (through equilibrium conditions) as

$$
g_{d}(x)=\frac{\phi_{1} w_{1} L_{1}}{\phi_{2} w_{2} L_{2}} \cdot x \quad \text { and } \quad g_{\phi}(x)=\left(\frac{P_{1}}{P_{2}}\right)^{-\varepsilon} .
$$

Thus, as mentioned above, parameters $\alpha$ and $\mu$ determine the strengths with which the relative manufacturing income and relative manufacturing price index affect relative utility, respectively, while parameters $d_{1}$ and $d_{2}$ affect the relative manufacturing income.

Since $V(x)$ is positively proportional to $\ln \omega$, the sign of $d V(x) / d L_{1}$ is the same as the sign of $d \ln \omega / L_{1}$. As shown in Appendix B.4, $\ln x$ is a decreasing function of $L_{1}$, and thus, using $d V(x) / d L_{1}=(d V(x) / d \ln x) \cdot\left(d \ln x / d L_{1}\right)$, we have that $d \omega / d L_{1}<0$ if and only if $d V(x) / d \ln x>0$. Thus, we can use function $V(x)$ for analysis of both uniqueness and stability of regular equilibria.

Equation $V(x)=0$ can readily be applied to establish existence of a regular equilibrium in the case with $\alpha \neq 1$. Indeed, function $V(x)$ is continuous and takes values at the opposite sides of 0 as $x \rightarrow 0$ and $x \rightarrow \infty .^{12}$ The analysis of existence of regular equilibria in the case with $\alpha=1$ is substantially more complicated and is described in detail in Section 2 of the Online Appendix.

Let us start the analysis of uniqueness and stability with the simple case of frictionless trade $\left(\phi_{1}=\phi_{2}=1\right)$. In this case, function $V(x)$ collapses to $V(x)=\ln G+(1-\alpha) \ln x$. If $\alpha \neq 1$ then we can explicitly find $x=G^{\frac{1}{\alpha-1}}$. Moreover, we also see that if $\alpha<1$ then $d V(x) / d \ln x>0$, while if $\alpha>1$ then $d V(x) / d \ln x<0$. If $\alpha=1$ and $G=1$ then any positive $x$ is a solution of $V(x)=0$ and $d V(x) / d \ln x=0$, while if $\alpha=1$ and $G \neq 1$, then equation $V(x)=0$ does not have positive solutions. These results give us the following proposition.

Proposition 2 (Regular equilibria: Frictionless trade). Assume that trade is frictionless, $\phi_{1}=\phi_{2}=1$.

(i) If $\alpha \neq 1$ then the economy has a unique regular equilibrium. If $\alpha<1$ then this equilibrium is globally stable, while if $\alpha>1$ then this equilibrium is unstable.

(ii) If $\alpha=1$ and $G=1$ then any regular allocation satisfying $L_{1}+L_{2}=\bar{L}$ is an equilibrium. Each of these equilibria is neither stable nor unstable. If $\alpha=1$ and $G \neq 1$ then the economy does not have regular equilibria.

In the rest of this section we focus on the case of costly trade: $\tau_{12} \tau_{21}>1$ or, equivalently, $\phi_{1} \phi_{2}<1$. One can easily verify that in the case with $\alpha \neq 1$ solving equation

\footnotetext{
${ }^{12}$ If $\alpha<1$ then $\lim _{x \rightarrow 0} V(x)=-\infty$ and $\lim _{x \rightarrow \infty} V(x)>+\infty$. If $\alpha>1$ then $\lim _{x \rightarrow 0} V(x)=+\infty$ and $\lim _{x \rightarrow \infty} V(x)=-\infty$.
} 
$V^{\prime}(x)=0$ for $x>0$ is equivalent to finding roots of a fourth-degree polynomial, and thus function $V(x)$ can have at most four extrema. This implies that equation $V(x)=0$ can have at most five solutions. Similarly, in the case with $\alpha=1$ solving equation $V^{\prime}(x)=0$ for $x>0$ is equivalent to finding roots of a quadratic polynomial, and thus function $V(x)$ can have at most two extrema, implying that equation $V(x)=0$ can have at most three solutions. Using this logic, we characterize formally the maximum number of regular equilibria in Proposition 6 in Appendix B.3.

The fact that $V^{\prime}(x)$ is at most a fourth-degree polynomial implies that, in principle, we can derive analytically necessary and sufficient conditions for uniqueness of regular equilibria for any $\alpha .{ }^{13}$ Moreover, this analysis can also yield analytical results on global or local stability of equilibria. However, the expressions would be complicated and devoid of insights. Instead, we will formulate several parsimonious sufficient conditions for uniqueness and global stability of regular equilibria. The proofs of these sufficiency results rely on establishing that under these conditions function $V(\cdot)$ is monotone.

The following proposition provides sufficient conditions for uniqueness and stability in the case of $\alpha \neq 1$ and costly trade. The proposition uses constants $\bar{\mu}_{a}, \bar{\mu}_{b}$, and $\overline{\bar{\mu}}$ that depend only on model parameters. Their definitions are provided in Appendix A. ${ }^{14}$

Proposition 3 (Regular equilibria: Sufficient conditions for uniqueness under costly trade and $\alpha \neq 1$ ). Assume that $\phi_{1} \phi_{2}<1$. The economy has a unique regular equilibrium in the following cases:

(i) $\alpha \leq 0$;

(ii) $0<\alpha<1$ and either of the following three conditions hold:

(ii.a) $d_{1} d_{2} \leq 1$ and $\mu \leq \bar{\mu}_{a^{\prime}}$;

(ii.b) $d_{1} d_{2}>1$ and $\mu \leq \bar{\mu}_{b}$;

(iii) $\alpha>1$ and either of the following three conditions hold:

(iii.a) $d_{1} d_{2} \leq 1$;

(iii.b) $d_{1} d_{2}>1$ and $\mu \geq \overline{\bar{\mu}}$.

In cases (i) and (ii) the regular equilibrium is generically globally stable, while in case (iii) it is unstable.

The proof of Proposition 3 is provided in Appendix B.5. In Section 4 of the Online Appendix we provide figures that give an idea on how much of the uniqueness area is

\footnotetext{
${ }^{13}$ Importantly, this is how we determine the number of regular equilibria for any combination of parameters to make all figures in this paper. See the discussion in Appendix B.3.

${ }^{14}$ For brevity of exposition, here and below we omit definitions of various functions of parameters. Appendix A contains all definitions.
} 
covered by sufficient conditions in part (ii) of Proposition 3. The qualification "generically" to the result on global stability in Proposition 3 is in the spirit of the literature on general equilibrium and means that under conditions in parts (i) and (ii) the relative utility $\omega$ is strictly decreasing in $L_{1}$ for all $L_{1}$ except, perhaps, for a finite number of points where $d \omega / d L_{1}=0 .{ }^{15}$ In Claim 1 in Appendix B.4 we prove an additional result concerning stability of equilibria: if an equilibrium is unique then it is generically locally stable for $\alpha \in(0,1)$ and generically unstable for $\alpha>1$.

Recall that the sufficient condition for uniqueness in Allen and Arkolakis (2014) is $\alpha \leq 0$. This is a strong condition that, for example, rules out agglomeration externalities if there are no congestion externalities. Part (i) of Proposition 3 extends this result to the economy with the agricultural sector and input-output loops. At the same time, Propositions 1 and 2 imply that the equilibrium is unique under frictionless trade even with $0<\alpha<1$. Proposition 3 extends this result to the case of low enough but strictly positive trade costs or low enough $\alpha .{ }^{16}$

Results of Proposition 3 give us the first hint to the role (for uniqueness) of agglomeration forces as well as the dispersion forces associated with terms of trade and agriculture, which we will study in detail in the next two sections. The strength of agglomeration forces (net of congestion) is captured by parameter $\chi$, while the strength of the term-oftrade dispersion force is given by $1 / \varepsilon$ and the strength of the agriculture dispersion force is governed by $\beta$. A high value of $1 / \varepsilon-$ implying a low value of $\alpha \equiv \chi \varepsilon$ given $\chi-$ means that as agents move to a region they suffer a larger terms-of-trade loss, which discourages concentration of production and tends to result in uniqueness. With low trade costs, thanks to the terms-of-trade dispersion force, agents do not tend to concentrate in one region, which pushes towards uniqueness of equilibrium. In Corollary 1 in Appendix B.5.1 we formally show that Proposition 3 implies that for $\alpha \in(0,1)$ there is a unique regular equilibrium if $\phi_{1} \phi_{2}$ is close enough to 1 or $\alpha$ is close enough to 0 . Turning to the dispersion force associated with presence of agriculture, in Corollary 2 in Appendix B.5.2 we show that there is a unique regular equilibrium if $\beta$ sufficiently low and there is no input-output loop.

The next proposition provides sufficient conditions for existence and uniqueness of a regular equilibrium in the case with $\alpha=1$ and costly trade. This proposition uses

\footnotetext{
${ }^{15}$ More precisely, as we show in the proof of Proposition 3, we can have $d \omega / d L_{1}=0$ only in part (ii.a) and only in one point (which might or might not exist depending on parameters).

${ }^{16}$ In Corollary 1 in Appendix B.5.1 we formally show that Proposition 3 implies that for $\alpha \in(0,1)$ there is a unique regular equilibrium if $\phi_{1} \phi_{2}$ is close enough to 1 or $\alpha$ is close enough to 0 .
} 
constants $\tilde{\phi}_{1}$ and $\tilde{\phi}_{2}$ defined in Appendix $\mathrm{A}$ as well as the following condition,

$$
\bar{\gamma} \zeta \bar{\beta} \phi_{2}^{\mu-1}+(1-\bar{\gamma} \zeta \bar{\beta}) \phi_{1} \phi_{2}^{\mu}<G<\left[\gamma \zeta \bar{\beta} \phi_{1}^{\mu-1}+(1-\gamma \zeta \bar{\beta}) \phi_{1}^{\mu} \phi_{2}\right]^{-1} .
$$

Proposition 4 (Regular equilibria: Sufficient conditions for existence and uniqueness under costly trade and $\alpha=1$ ). Assume that $\alpha=1$ and $\phi_{1} \phi_{2}<1$. Suppose that one of the following conditions holds:

(a) $\bar{\beta}=0$, or $\gamma=0$, or $\gamma=1$;

(b) $\mu \geq 1$;

(c) $\mu<1$ and either $\phi_{1} \geq \tilde{\phi}_{1}$ or $\phi_{2} \geq \tilde{\phi}_{2}$.

If, in addition to that, condition (14) holds, then the economy has a unique regular equilibrium, which is generically unstable. If, on the other hand, condition (14) does not hold, then the economy does not have regular equilibria.

The proof of Proposition 4 is provided in Appendix B.6. There we show a more precise result in terms of stability: if condition (14) holds then the equilibrium is unstable in cases (a) and (b) as well as in the case (c) with $\mu<1$ if either $\phi_{1}>\tilde{\phi}_{1}$ or $\phi_{2}>\tilde{\phi}_{2}$. If in the case (c) we have $\phi_{1}=\tilde{\phi}_{1}$ or $\phi_{2}=\tilde{\phi}_{2}$ then - depending on parameter values - it can happen that $d \omega / d L_{1}=0$.

Conditions in Proposition 4 do not cover the case in which $\beta \in(0,1), \gamma \in(0,1), \mu<1$, and $\phi_{1}<\tilde{\phi}_{1}$ and $\phi_{2}<\tilde{\phi}_{2}$. This case is much more difficult, but it is possible to provide useful qualitative results in this case. ${ }^{17}$ Proposition 1 in Section 1 of the Online Appendix establishes that there exists a unique (locally stable) regular equilibrium for large enough trade costs. Also, we show that there can be a unique (unstable) regular equilibrium for all "not too large" trade costs such that $\phi_{1}<\tilde{\phi}_{1}$ or $\phi_{2}<\tilde{\phi}_{2}$. Finally, we show that for all "intermediate" levels of trade costs, the economy can have up to three regular equilibria: two unstable and one locally stable.

\section{No Agricultural Sector}

In this section we consider a version of the economy of Section 2 with no agricultural sector, $\beta=1$. This corresponds to a two-region version of the setup in Allen and Arkolakis (2014) extended to allow for an input-output loop. In what follows, we call this version of the setup the "AA economy".

\footnotetext{
${ }^{17}$ Recall that, using function $V$, we can provide the full characterization of regular equilibria for any $\alpha$. As an example of how one can do this, we provide such characterization for $\alpha=1$ in Section 2 of the Online Appendix. However, this characterization is devoid of insights.
} 


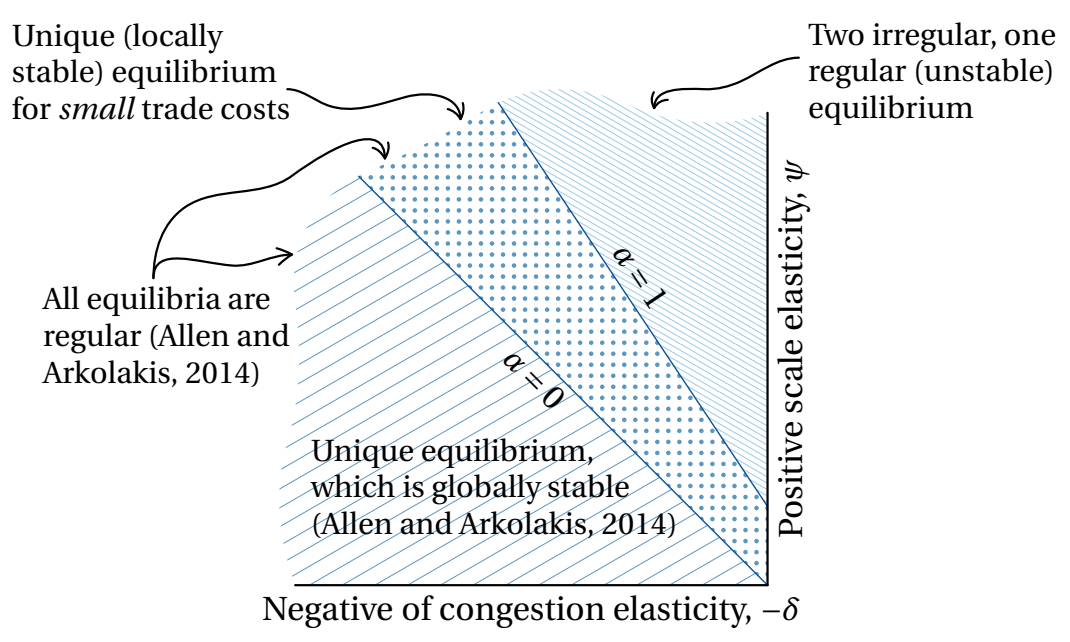

Figure 1: Equilibria in the AA economy for a fixed $\varepsilon$ and $\zeta$.

We can collect different parts of Propositions 1-4 to provide a complete characterization of equilibria of the AA economy in the cases with $\alpha \leq 0$ and $\alpha \geq 1$. It helps to refer to Figure 1, which shows the areas with a unique regular equilibrium as well as areas with irregular equilibria in the space of $(\delta, \psi)$ for given values of $\varepsilon$ and $\zeta$.

If $\alpha \leq 0$ then the AA economy has a unique equilibrium and this equilibrium is regular and globally stable, while if $\alpha>1$ then the AA economy has two irregular equilibria and a unique regular equilibrium, which is unstable. ${ }^{18}$ The case with $\alpha=1$ is a bit more involved. We get that allocation $L_{1}=\bar{L}$ and $L_{2}=0$ is an equilibrium if and only if $\phi_{1} \phi_{2}^{\mu} \leq G$, while allocation $L_{1}=0$ and $L_{2}=\bar{L}$ is an equilibrium if and only if $\left(\phi_{1}^{\mu} \phi_{2}\right)^{-1} \geq$ G. Also, if $\phi_{1} \phi_{2}<1$ and $\phi_{1} \phi_{2}^{\mu}<G<\left(\phi_{1}^{\mu} \phi_{2}\right)^{-1}$ then there is a single regular (unstable) equilibrium, while if $\phi_{1}=\phi_{2}=1$ and $G=1$ then any regular allocation satisfying $L_{1}+L_{2}=\bar{L}$ is an equilibrium, which is neither stable nor unstable. And there are no regular equilibria in all other cases when $\alpha=1$.

We know from Proposition 1 that all equilibria in the AA economy are regular when $0<\alpha<1$. Part (ii.a) of Proposition $3, \mu \leq \bar{\mu}_{a^{\prime}}$ gives us only sufficient conditions for uniqueness of regular equilibria of the AA economy in this case. In the next proposition we provide a complete characterization of regular equilibria when $0<\alpha<1$, using $\bar{\mu}_{a}$ as well as constants $\Gamma_{1}$ and $\Gamma_{2}$ that depend on $\mu, \alpha, \phi_{1}, \phi_{2}$, and $G$ and that are defined in Appendix A.

Proposition 5 (AA economy: Complete characterization of equilibria for $0<\alpha<1$ ). Assume that $\beta=1,0<\alpha<1$, and $\phi_{1} \leq 1$ and $\phi_{2} \leq 1$. Then any equilibrium is regular.

(a) There is a unique equilibrium in the following cases:

\footnotetext{
${ }^{18}$ To get the result for $\alpha>1$, note that $d_{1}=\phi_{1}$ and $d_{2}=\phi_{2}$ when $\beta=1$. Thus, we can apply part (iii.a) of Proposition 3.
} 
(a.1) $\mu \leq \bar{\mu}_{a}$ (or equivalently $\sqrt{\phi_{1} \phi_{2}} \geq \frac{\mu+2 \alpha-1}{\mu+1}$ );

(a.2) $\mu>\bar{\mu}_{a}$ and either $\Gamma_{1}<1$ or $\Gamma_{2}>1$.

In case (a.1) the equilibrium is generically globally stable, while in case (a.2) the equilibrium is generically locally stable.

(b) There are two equilibria if $\mu>\bar{\mu}_{a}$ and either $\Gamma_{1}=1$ or $\Gamma_{2}=1$. One equilibrium is locally stable, the other equilibrium is neither stable nor unstable.

(c) There are three equilibria if $\mu>\bar{\mu}_{a}$ and $\Gamma_{1}>1$ and $\Gamma_{2}<1$. Two equilibria are locally stable, and one equilibrium is unstable.

The proof of Proposition 5 is provided in Appendix C. One noteworthy implication of this proposition is that the presence of an input-output loop, as captured by $\zeta<1$, affects the set of equilibria in the AA economy only through parameters $\chi=\frac{\psi-\zeta \delta}{1+\delta}$ and $G=\left(\bar{u}_{1} / \bar{u}_{2}\right)^{\alpha+\zeta \varepsilon}\left(\bar{A}_{1} / \bar{A}_{2}\right)^{\varepsilon}$. Consider the case with no asymmetries in amenities $\left(\bar{u}_{1}=\right.$ $\left.\bar{u}_{2}\right)$, which implies that any regular equilibrium entails equalized real wages across the two regions, $w_{1} L_{1}^{-\delta} / P_{1}=w_{2} L_{2}^{-\delta} / P_{2}$. The definition of $x$ then implies that any regular equilibrium entails

$$
x=\left(\frac{\bar{A}_{1}}{\bar{A}_{2}}\right)^{-\varepsilon}\left(\frac{P_{1}}{P_{2}}\right)^{\varepsilon \mu}\left(\frac{w_{1} L_{1}}{w_{2} L_{2}}\right)^{-\alpha} .
$$

The relative price $P_{1} / P_{2}$ depends on $\zeta$ only through $x$, which is one reason why $x$ was introduced in the first place - see expressions (13). Similarly, the relative manufacturing output, $w_{1} L_{1} / w_{2} L_{2}$, also depends on $\zeta$ only through $x$ if $\beta=1 .{ }^{19}$ Thus, if there are no congestion externalities $(\delta=0)$ then real-wage equalization across the regions implies that the presence of an input-output loop does not affect the set of equilibria (although $\zeta$ does affect labor allocations in each particular equilibrium). With congestion externalities $(\delta>0)$, smaller values of $\zeta$ lead to higher values of $\chi$, which, as discussed below, makes multiplicity more likely.

Let us now explore the implications of Proposition 5 for the way in which different model parameters affect the set of equilibria in the AA economy. Figure 2 illustrates the role of parameters $\alpha, \mu$, and $\phi$ for the symmetric economy with $G=1$ and $\phi \equiv \phi_{1}=\phi_{2} \cdot{ }^{20}$ Figure 2 shows that for each fixed level of trade costs, lower values of $\alpha$ or $\mu$ tend to result in uniqueness, while higher values of $\alpha$ and $\mu$ tend to result in multiplicity. To understand this outcome, first note that in the symmetric case illustrated in Figure 2, the symmetric allocation associated with $x=1$ is always an equilibrium. In this equilibrium each region

\footnotetext{
${ }^{19}$ Formally, this follows from expression (6) and the fact that relative trade shares $\lambda_{12} / \lambda_{21}$ depend on $\zeta$ only through $x$ - again, one of the reasons why $x$ was introduced in the first place.

${ }^{20}$ Observe that with $\beta=1$ we have $\mu=\chi+1$ and so $\mu>1$ for $\alpha \in(0,1)$. Also, a typical value of $\varepsilon=5$ would imply $\mu<1.2$ for $\alpha \in(0,1)$. At the same time, we can have any value of $\mu>1$ by varying $\varepsilon$, which implies that $\mu^{-1} \in(0,1)$ for $\alpha \in(0,1)$.
} 


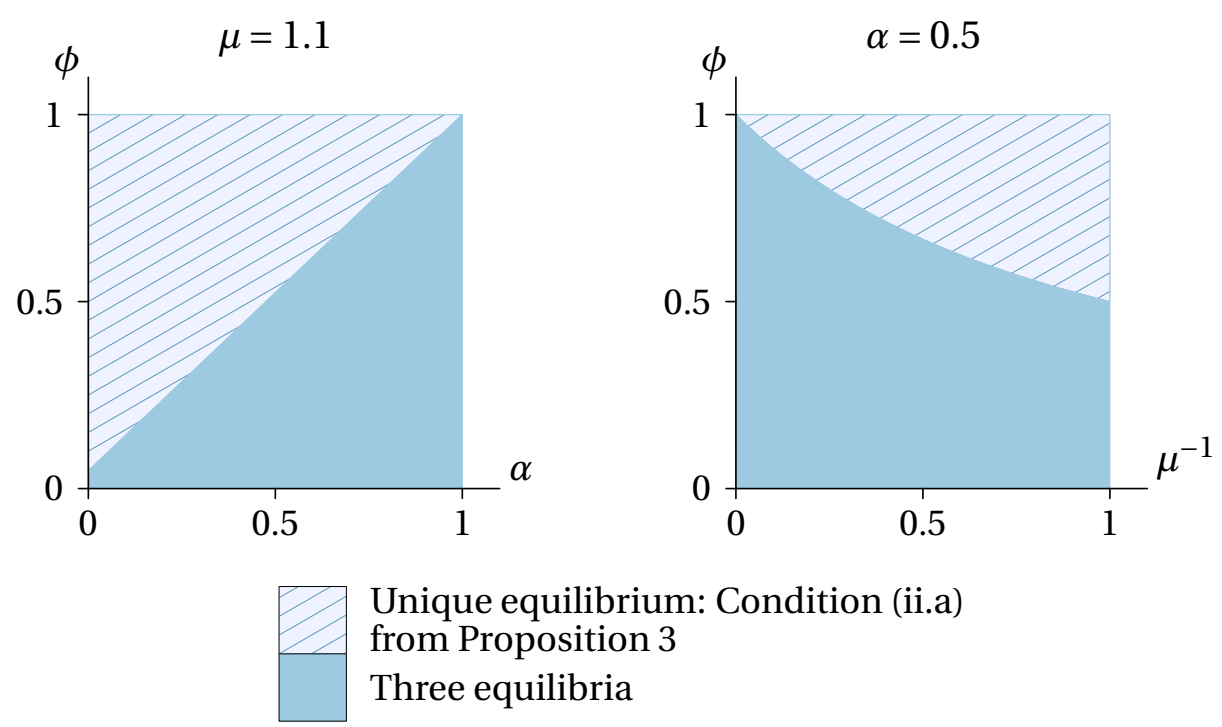

Figure 2: Uniqueness/multiplicity areas in Proposition 5 for AA economy: $\phi \equiv$ $\phi_{1}=\phi_{2}$ and $G=1$. Left-hand side: varying $\alpha$ and $\phi$ for $\mu=1.1$. Right-hand side: varying $\mu^{-1}$ and $\phi$ for $\alpha=0.5$. The boundary between the sets with unique and three equilibria has two equilibria.

gets half of the manufacturing labor force. Then, because of symmetry, to understand how multiplicity arises, it is enough to consider equilibria with $x<1$, where production of the manufacturing good in region 1 is relatively cheaper and this region gets relatively more of the manufacturing workers.

Consider the case with $\alpha=0$ and $\mu=1$. Using $\left(P_{1} / P_{2}\right)^{-\varepsilon}=(1+\phi x) /(\phi+x)$, we can write (15) as

$$
x=\frac{\phi+x}{1+\phi x},
$$

where we assumed symmetry in exogenous manufacturing productivity, $\bar{A}_{1}=\bar{A}_{2}$. It is easy to verify that for $x<1$ the right-hand side of the above expression is larger than $x$, and thus $x=1$ is the only solution to this equation. Dropping for a moment the relationship between $\alpha$ and $\mu$, consider the case with $\alpha=0$ and $\mu>1$. Then (15) turns into

$$
x=\left(\frac{\phi+x}{1+\phi x}\right)^{\mu} .
$$

One can easily check that for all $\mu$ large enough, the right-hand side of this equation is smaller than $x$ for $x$ close to 1 , and is larger than $x$ for $x$ close to 0 . Thus, there is a solution to the above equation for some $x<1$. Intuitively, with $\mu=1$, the relative price index associated with a non-symmetric labor allocation is always larger than the relative cost of the manufacturing good, which leads to uniqueness of equilibrium. Having $\mu>1$ 
magnifies the differences in price indices across the regions arising in asymmetric allocations, which leads to a possibility of multiple equilibria where manufacturing production is concentrated in one of the regions.

The impact of parameter $\mu$ described above is not surprising. As mentioned in Section 3, parameter $\mu$ captures the strength of the net agglomeration force in manufacturing relative to the dispersion force associated with the agriculture sector. With no agriculture, $\mu$ is just given by $\chi+1$, which captures agglomeration forces working through the manufacturing price index, and hence it is intuitive that increasing the strength of the agglomeration force leads to multiplicity. In turn, increasing $\alpha$ leads to the possibility of multiple equilibria by magnifying differences in manufacturing labor incomes across regions given by $w_{1} L_{1} / w_{2} L_{2}$. For given $\mu$, a higher value of $\alpha$ is associated with either stronger net agglomeration force or weaker terms-of-trade dispersion force given by $1 / \varepsilon$. A high value of $1 / \varepsilon$ means that as agents move to a region they suffer a larger terms of trade loss, which discourages concentration of production and tends to result in uniqueness.

Returning to Figure 2, we also see that for each fixed $\alpha$ and $\mu$, lower trade costs tend to result in uniqueness, while higher trade costs tend to result in multiplicity. With low trade costs, thanks to the terms-of-trade dispersion force, agents do not tend to concentrate in one region, which results in uniqueness of an equilibrium. High trade costs weaken this dispersion force by limiting trade, thereby making multiple equilibria more likely.

Figure 2 also shows that condition $\mu \leq \bar{\mu}_{a}$ in part (a.1) of Proposition $5-$ which is the same as the condition in part (ii.a) of Proposition 3 - covers the entire uniqueness area in the symmetric case. In Corollary 1 in Section 3 of the Online Appendix we formally establish that in the symmetric case of the AA economy with $0<\alpha<1$ condition $\mu \leq \bar{\mu}_{a}$ is both necessary and sufficient for uniqueness. For $\beta=1$ and $\alpha \in(0,1)$ this condition can equivalently be written as $\phi \geq \frac{\mu+2 \alpha-1}{\mu+1} \in(0,1)$, where $\phi \equiv \phi_{1}=\phi_{2}$. Thus, in the symmetric case, as we lower $\phi$ from 1 to 0 (i.e., raise trade costs from 1 to $\infty$ ), we go from the region of uniqueness to the region of multiplicity, with the threshold given by $\frac{\mu+2 \alpha-1}{\mu+1}$. It turns out that this is a general property of the AA economy (allowing for asymmetries), which we formally establish in Proposition 2 in Section 3 of the Online Appendix. Specifically, there we show that in the AA economy with $0<\alpha<1$ for any fixed ratio $c \equiv \phi_{1} / \phi_{2}$ there exists a threshold $\tilde{\phi}_{2}(c)>0$ such that the equilibrium is unique if $\phi_{2}>\tilde{\tilde{\phi}}_{2}(c)$, and there are three equilibria if $\phi_{2}<\tilde{\tilde{\phi}}_{2}(c) .{ }^{21}$ For some parameter

\footnotetext{
${ }^{21}$ Generically there are two equilibria for $\phi_{2}=\tilde{\tilde{\phi}}_{2}(c)$. The symmetric setup is an example of a nongeneric case when there is a unique equilibrium at the threshold. Also, generically there is no explicit expression for the threshold $\tilde{\tilde{\phi}}_{2}(\mathrm{c})$ : this threshold can be found from conditions (a)-(c) of Proposition 5 as an implicit function of model parameters.
} 
combinations we can have $\tilde{\tilde{\phi}}_{2}(c)>1$, in which case there are three equilibria for all levels of positive trade costs. It is worth emphasizing that the threshold $\tilde{\tilde{\phi}}_{2}(c)$ exists for a fixed ratio $\phi_{1} / \phi_{2}$, and thus as we lower $\phi_{2}$ from 1 to 0 we also change $\phi_{1}$ in the same proportion. In contrast to this, if we keep, say, $\phi_{1}$ fixed and lower $\phi_{2}$ from 1 to 0 , we can have intermittent regions of one and three equilibria.

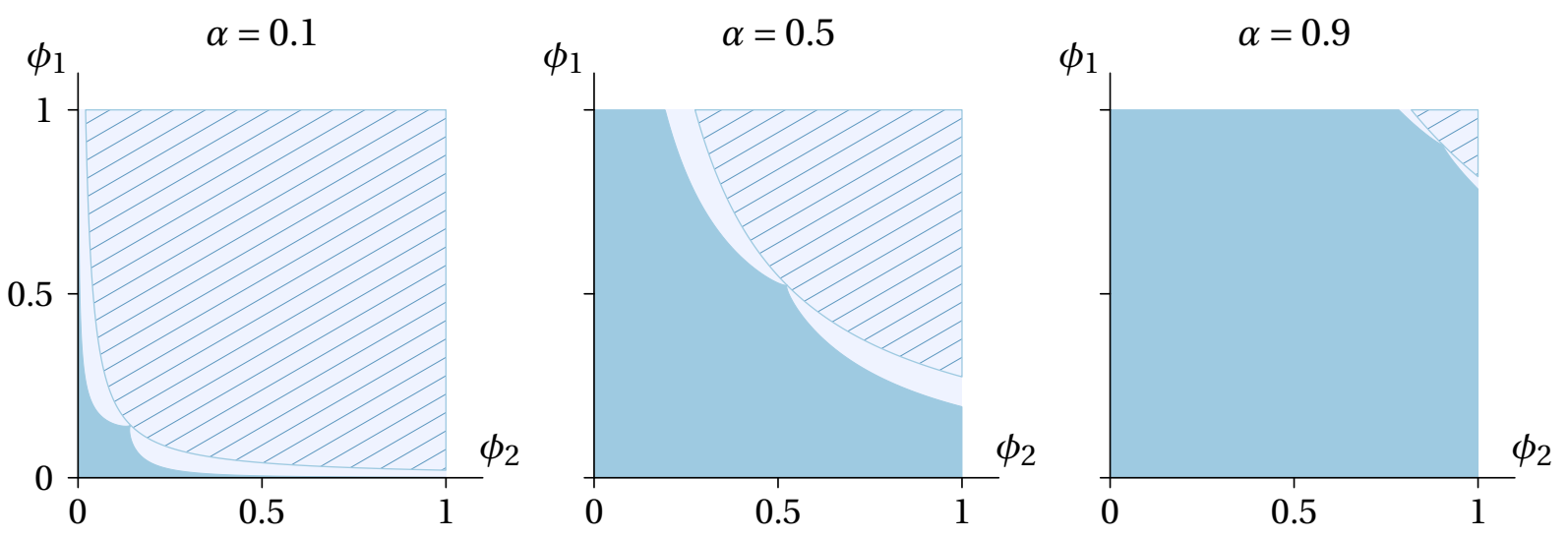

(a) Varying $\phi_{1}$ and $\phi_{2}$ with $G=1$.
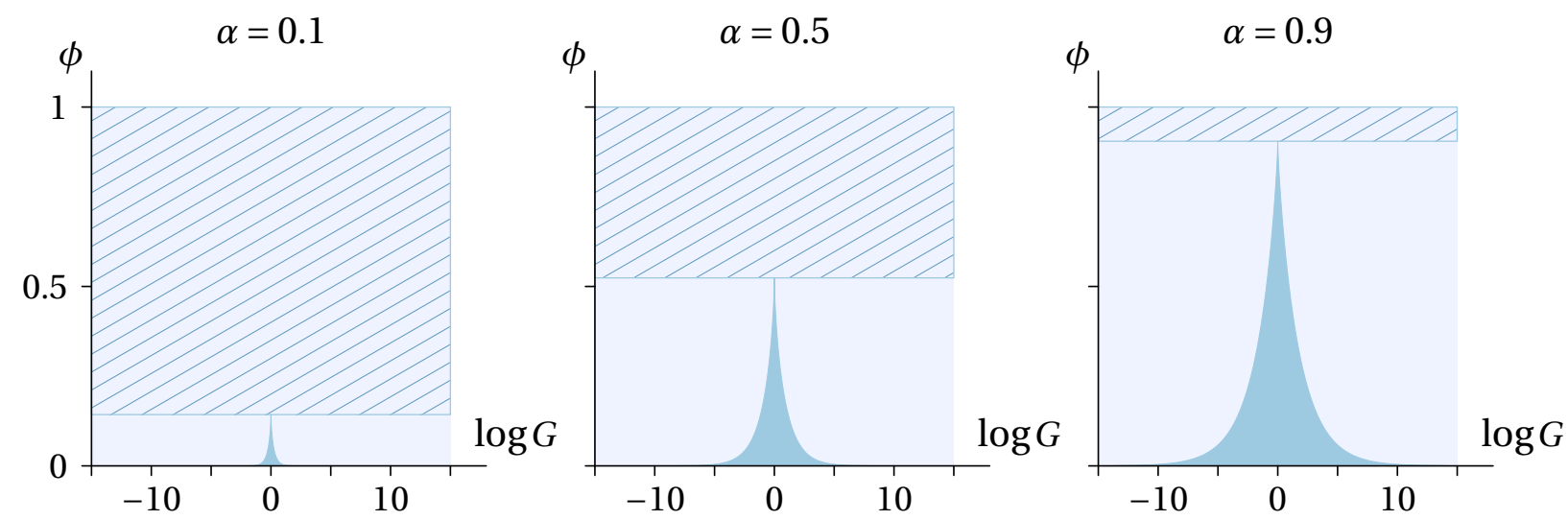

(b) Varying $\phi$ and $G$ with $\phi \equiv \phi_{1}=\phi_{2}$.

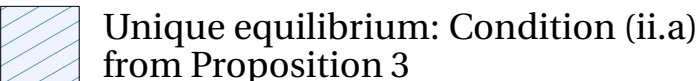
from Proposition 3

Unique equilibrium: Not covered by condition (ii.a) of Proposition 3

Three equilibria

Figure 3: Uniqueness/multiplicity areas in Proposition 5 for AA economy: $0<\alpha<1$ and $\mu=1.1$.

Let us now turn to Figure 3, which illustrates several points. First, without symmetry condition $\mu \leq \bar{\mu}_{a}$ is no longer sufficient for uniqueness. Second, larger values of $\alpha$ are more likely to result in multiplicity (the point that we made earlier). Finally, Figure 3 shows how asymmetries in trade costs and in productivities or amenities of regions impact uniqueness/multiplicity. 
We see from panel (b) of Figure 3 that asymmetries in productivities or amenities across regions tend to result in uniqueness. Intuitively, multiplicity of equilibria in the symmetric setup arises exactly because all fundamental parameters of regions are the same, which - due to the economies of scale - creates indeterminacy of the outcome: manufacturing production can happen to be concentrated in one region or the other. As we make regions dissimilar in their productivities or amenities, the region with the more favorable characteristic is more likely to become an industrial hub with concentrated manufacturing production, which reduces indeterminacy of the outcome and leads to uniqueness of equilibrium.

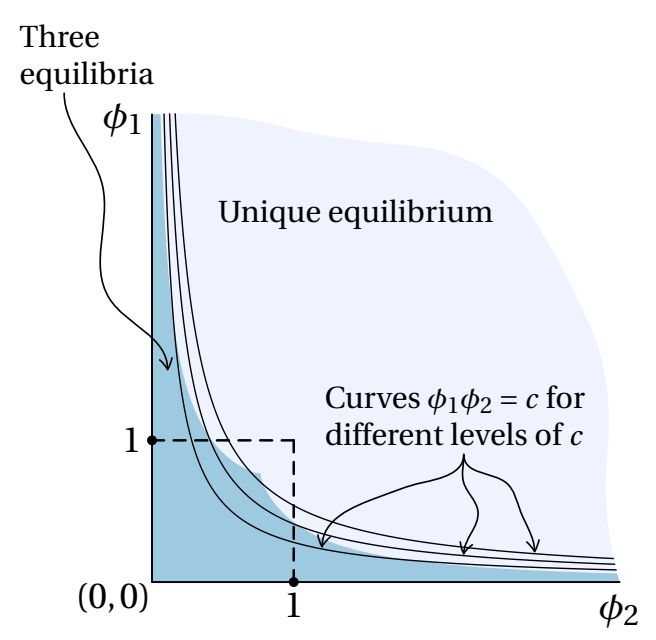

Figure 4: Illustration to Proposition 5 for AA economy: $0<\alpha<1$ and $G=1$. Asymmetries in trade costs.

Let us now consider asymmetries in trade costs. In order to isolate the effect of asymmetries in trade costs from the effect of the size of trade costs, we focus on combinations of $\phi_{1}$ and $\phi_{2}$ such that the geometric mean of trade freeness, $\left(\phi_{1} \phi_{2}\right)^{1 / 2}$, is constant, $\phi_{1} \phi_{2}=c$. Along such a curve, the more the ratio $\phi_{1} / \phi_{2}$ is different from 1 , the larger asymmetries in trade costs are. In Figure 4 we show areas with one and three equilibria in the $\left(\phi_{1}, \phi_{2}\right)$ space - just as in panel (a) of Figure 3. On top of that, we depict curves $\phi_{1} \phi_{2}=c$ for three levels of $c$. Also, for illustration purposes, we drop the assumption that $\phi_{1} \leq 1$ and $\phi_{2} \leq 1$. Figure 4 shows that increasing asymmetry in trade costs tends to result in uniqueness. Intuitively, for the same geometric mean of trade costs, asymmetry in trade costs creates incentives to concentrate manufacturing production in the region with high cost of importing and low cost of exporting, which leads to uniqueness. The same outcome is also evident from panel (a) of Figure 3. However, as Figure 3 shows, imposing the restriction $\phi_{1} \leq 1$ and $\phi_{2} \leq 1$ limits the effect of asymmetries on uniqueness: for example, with 
$\alpha=0.9$ the model has three equilibria for most combinations of trade costs.

\section{Symmetric Regions}

In Section 4 we used a special case with $\beta=1$ to study the role of trade costs and parameters $\alpha$ and $\mu$ in determining the set of equilibria. In the current section we turn to the case of symmetric regions to study the role of parameter $\beta$. The case with symmetric regions is formally given by parameter restrictions $\gamma=0.5, G=1$, and $\phi_{1}=\phi_{2} \equiv \phi$. In this case $x=1$ is always a solution to $V(x)=0$, which corresponds to the outcome where each region gets half of the manufacturing labor force. ${ }^{22}$ Compared to the case with $\beta=1$, the patterns of equilibria in the case with $\beta \neq 1$ are more complex: there can be five equilibria for some parameter combinations, and there can be a unique equilibrium for all large enough trade costs.

As evident from our key equation $V(x)=0$, parameter $\beta$ impacts the set of equilibria only through parameters $\mu=\beta \chi+1-\zeta \bar{\beta}$ and $d_{1}=d_{2}=0.5 \zeta \bar{\beta} \phi^{-1}+(1-0.5 \zeta \bar{\beta}) \phi$. By fixing $\zeta \bar{\beta}$ and changing $\mu$ (which entails changing $\beta \chi$ ), we can analyze the impact of parameter $\beta$ on the set of equilibria through $\mu$ only, which we do first. ${ }^{23}$ Similar to the case with $\beta=1$ discussed in Section 4 , increasing $\mu$ in the case with $\beta \neq 1$ magnifies differences in consumer prices across locations, which potentially leads to multiplicity of equilibria.

To set the stage, let us first consider the relatively well-studied Krugman case $(\alpha=1)$ illustrated in Figure 5. In this figure, we fix $\zeta \bar{\beta}=0.5$ and depict the sets and patterns of equilibria for different values of $\mu$ and $\phi .{ }^{24}$ In the symmetric Krugman case, Propositions 1,2 , and 4 can readily be applied to formally establish the patterns of equilibria illustrated in Figure 5. Observe that condition $\mu<1$ combined with $\alpha=1$ is equivalent to $\beta<\frac{\zeta \varepsilon}{\zeta \varepsilon+1}$, which is an extension of the so-called "no-black-hole condition" from Krugman (1991) to the case with an input-output loop. Thus, as is evident from Figure 5, our results naturally extend what we know about the original Krugman (1991) model

\footnotetext{
${ }^{22}$ In Section 4 of the Online Appendix we explore the role of asymmetries for the case with $0<\alpha<1$.

${ }^{23}$ Having $\zeta \bar{\beta}$ fixed still allows for changes in $\beta$ if they are accompanied by corresponding changes in $\zeta$.

${ }^{24}$ In Figure 5, we show equilibria maps in two alternative spaces: $\left(\mu^{-1}, \phi^{-1}\right)$ and $\left(\mu^{-1}, \phi\right)$. The focus of this figure is the $\left(\mu^{-1}, \phi^{-1}\right)$ space, for which we illustrate two points in the $\left(\phi^{-1}, L_{1}\right)$ coordinates. The figure in the $\left(\mu^{-1}, \phi\right)$ space allows us to zoom-in on the area close to the frictionless trade and grasp the pattern of equilibria in the whole space of trade costs. This proves to be especially useful when $\alpha<1$, as is made evident in Figure 6. In other sections, we show equilibria maps only for the trade freeness parameters $\phi_{i}$. Also, we consider $\phi^{-1}=\tau^{\varepsilon}$ instead of the trade costs $\tau$ because changing $\mu$ can be achieved by changing $\varepsilon$, and it is the composite parameter $\phi$ that is relevant for the uniqueness/multiplicity outcome rather than simply $\tau$.
} 


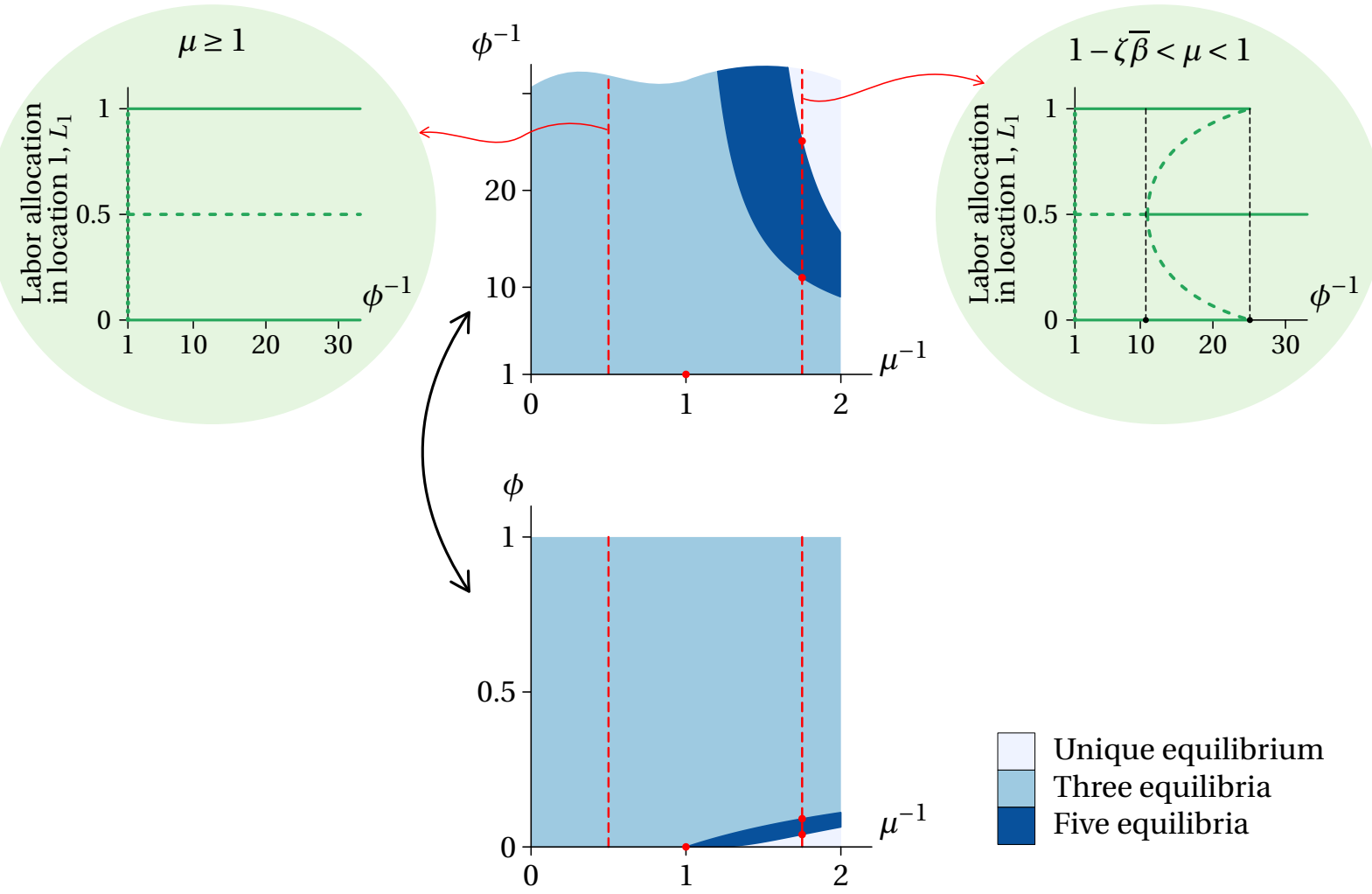

Figure 5: Symmetric Krugman case, $\alpha=1, \zeta \bar{\beta}=0.5$, and $\bar{L}=1$. Both regular and irregular equilibria are counted in the figures. Solid lines on the labor allocation pictures indicate stable equilibria, while dashed lines indicate unstable equilibria. When $\phi^{-1}=1$, equilibria are neither stable nor unstable.

(see, for example, Combes et al., 2008) to the case with an input-output loop: under the black-hole condition the core-periphery structure is always a possibility, and the symmetric equilibrium is the third possible equilibrium, which is unstable. If, on the other hand, the no-black-hole condition holds, then the picture that shows labor allocation patterns for each value of trade costs is the "tomahawk diagram". In this case there is a unique regular equilibrium if trade costs are low (resulting in an unstable equilibrium) or high (resulting in a stable equilibrium), while intermediate levels of trade costs result in three regular equilibria (one stable and two unstable). At the same time, irregular equilibria exist only for low and intermediate levels of trade costs.

Let us now consider the case with $\alpha \in(0,1)$ illustrated in Figure 6. In this figure we still keep $\zeta \bar{\beta}=0.5$ and show the sets and patterns of equilibria for $\alpha=0.7$ and for different values of $\mu$ and $\phi$. As we can see from Figure 6, relaxing the assumption $\alpha=1$ of the Krugman case significantly changes the set of equilibrium labor allocations. First, as established in Proposition 1, all equilibria are regular. Second, with $\alpha \in(0,1)$ there are four different patterns of labor allocations depending on the value of $\mu$. For 


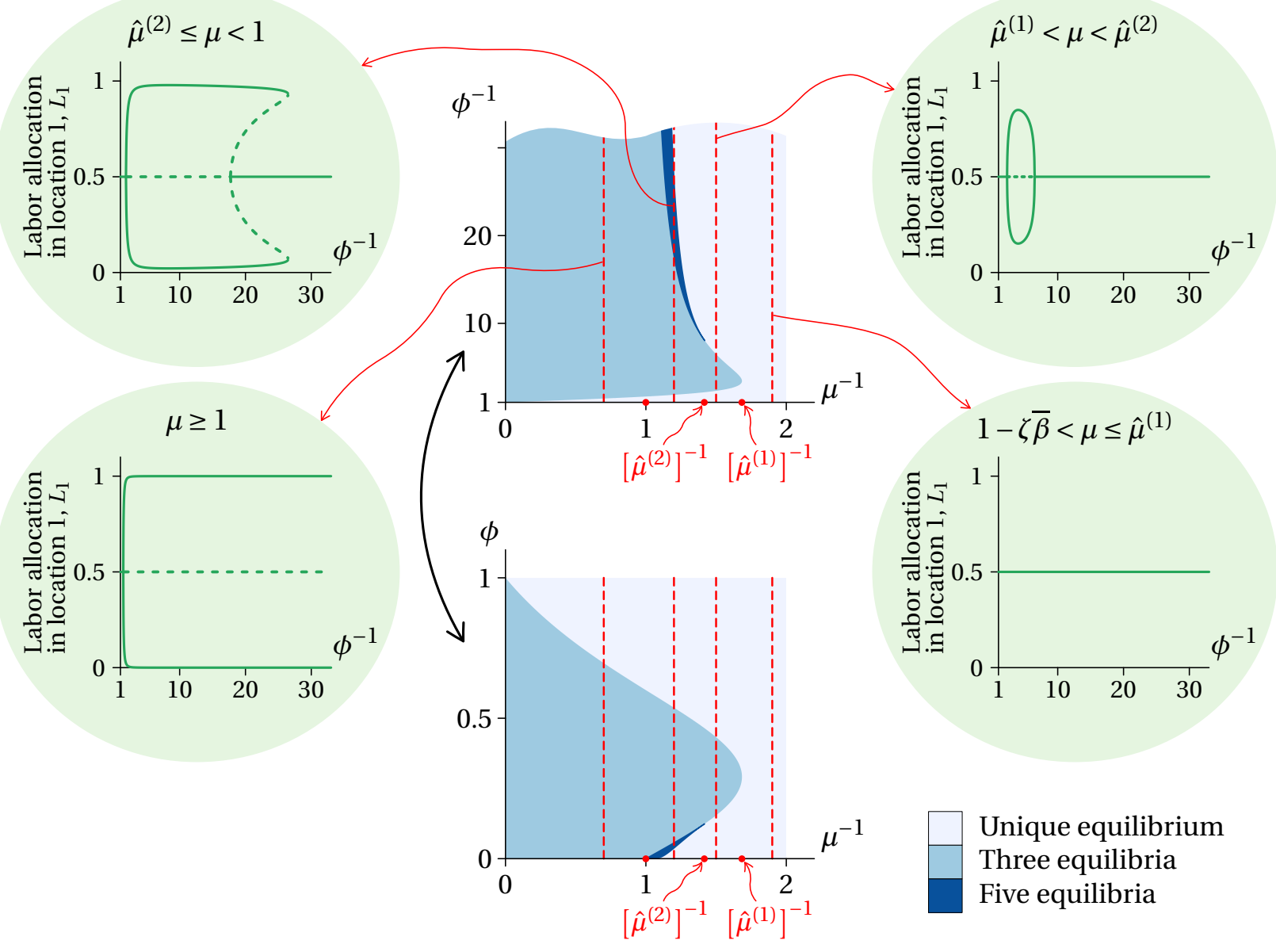

Figure 6: Symmetric case with $\alpha=0.7, \zeta \bar{\beta}=0.5$, and $\bar{L}=1$. All equilibria are regular. Solid lines on the labor allocation pictures indicate stable equilibria, while dashed lines indicate unstable equilibria.

low values of $\mu$ the equilibrium is unique. For lower to medium values of $\mu$ the pattern of labor allocations is a displaced ellipse. For medium to higher values of $\mu$ the pattern of labor allocations is a displaced tomahawk. And for high values of $\mu$ the pattern of labor allocations is a pitchfork..$^{25}$

In Appendix D we show why $\mu \geq 1$ is a necessary condition for the pitchfork pattern in the symmetric case with $\alpha \in(0,1)$. Observe that the pitchfork pattern in Figure 6 resembles the pattern of equilibria in Figure 5 for $\mu \geq 1$ (the important differences are that in Figure 6 all equilibria are regular and the equilibrium is unique for all small enough trade costs). Thus, similar to the case with $\alpha=1$, we can think of condition $\mu<1$ as the no-black-hole condition in the case with $\alpha \in(0,1)$. For $\alpha \in(0,1)$ the black-hole condition means that for all large enough trade costs the core-periphery structure is always

\footnotetext{
${ }^{25}$ As we discussed in footnote 16 , with $0<\alpha<1$ the equilibrium is always unique for low enough trade costs. This creates the "displaced" patterns in Figure 6.
} 
a possibility, and the symmetric equilibrium is the third possible equilibrium, which is unstable.

Besides the patterns of equilibria, another important message of Figures 5 and 6 is that, for a fixed level of trade costs, lower values of $\mu$ (higher values of $\mu^{-1}$ ) tend to result in a unique equilibrium, while higher values of $\mu$ tend to result in multiple equilibria. Similarly to the case with $\beta=1$ discussed in Section 4 , with $\beta \neq 1$ parameter $\mu$ determines how the difference in manufacturing price indices across regions impacts the relative utility. Higher values of $\mu$ magnify the difference and tend to result in multiplicity, while lower values of $\mu$ do the opposite. Lower values of $\mu$ are associated with lower values of $\beta$. In turn, lower values of $\beta$ imply a lower share of the manufacturing good in consumption, thus limiting the role of the relative manufacturing price index (and therefore of the agglomeration force) in leading to agglomeration and pushing towards multiplicity.

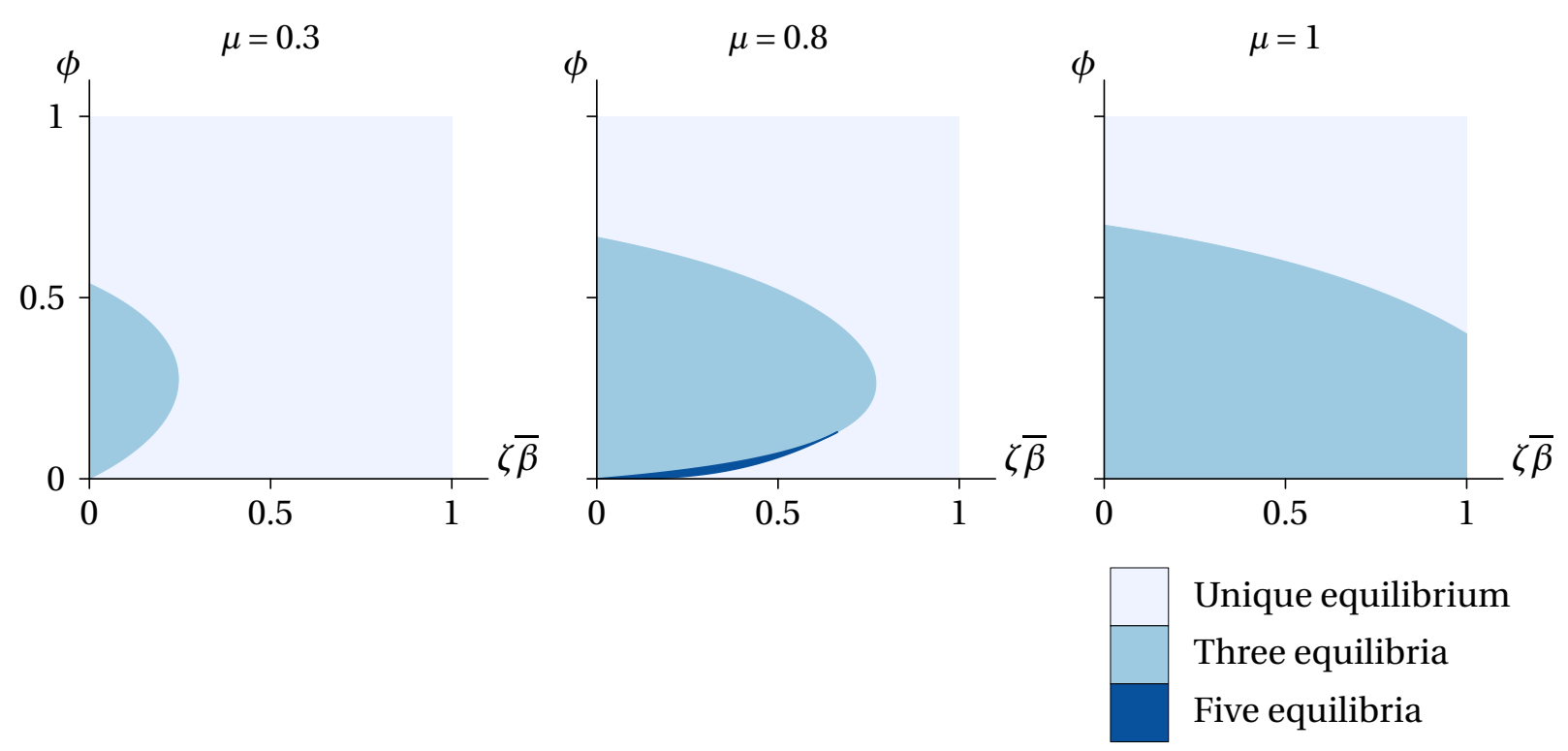

Figure 7: Symmetric case with $\alpha=0.7$. Equilibria sets for different values of $\mu$ in the $(\zeta \bar{\beta}, \phi)$ space. The parameter restriction $\mu>1-\zeta \bar{\beta}$ is dropped for illustration purposes.

Now consider the impact of parameter $\beta$ on the set of equilibria through $d \equiv d_{1}=d_{2}$. In Figure 7 , we depict the sets of equilibria in the space $(\zeta \bar{\beta}, \phi)$ for $\mu=0.3,0.8$, and $1 .^{26}$ We see from Figure 7 that for a fixed $\mu$, lower values of $\zeta \bar{\beta}$ tend to result in multiple equilibria, while higher values of $\zeta \bar{\beta}$ tend to result in a unique equilibrium. For a fixed $\zeta$, higher values of $\zeta \bar{\beta}$ are associated with lower values of $\beta$ implying a lower share of the manufacturing aggregate in consumption and a higher share of the agricultural good. Thus, lower values of $\beta$ tend to increase demand for the agricultural labor relative to the

\footnotetext{
${ }^{26}$ Pictures for $\mu<0.308,0.308<\mu<1$, and $\mu>1$ are qualitatively similar to the pictures with $\mu=0.3$, $\mu=0.8$, and $\mu=1$, correspondingly.
} 
manufacturing labor, which leads to higher agricultural wages relative to the manufacturing wages. This is reflected in larger values of $d$ and makes the manufacturing labor income more equalized across the regions, leading to uniqueness. ${ }^{27}$

Let us now consider the outcome with respect to trade costs observed in all Figures 57. First, similarly to the case with $\beta=1$ discussed in Section 4 , with $\beta<1$ small trade costs tend to result in uniqueness due to the terms-of-trade dispersion force. Second, differently from the case with $\beta=1$, for $\beta<1$ large trade costs can also result in a unique equilibrium. This happens due to the agricultural-sector dispersion force. Our assumption that $\beta>0$ implies that there is always a nonzero demand for the manufacturing aggregate in each region because of the presence of the immobile agricultural workers-consumers located in this region. Since production of the manufacturing aggregate requires differentiated manufactured goods from both regions, both regions have to have some amount of the differentiated manufactured good production. Large trade costs imply that a large proportion of the manufactured good is lost during transportation, requiring a larger amount of this good to be produced in each region. This discourages concentration of production in one region and leads to uniqueness. Another way to see this outcome is through our equation $V(x)=0$ : larger trade costs increase $d$, making incomes more equalized across the regions and leading to uniqueness.

Finally, let us consider the effect of the input-output loop on the set of equilibria. Compared to the case with $\beta=1$ discussed in Section 4 , when $\beta<1$ parameter $\zeta$ affects $\mu$ and $d$ independently from affecting $\alpha$ and G. The effect of $\zeta$ through $\alpha$ on the set of equilibria in the case with $\beta<1$ is the same as in the case with $\beta=1$ : in the presence of congestion effects, lower values of $\zeta$ (implying a higher share of the manufacturing composite in the production of the manufactured good) increase $\alpha$, which tends to result in multiplicity. The independent effect of $\zeta$ on $\mu$ works in an intuitive way: lower values of $\zeta$ imply lower values of $\zeta \bar{\beta}$, which increases $\mu$ and tends to result in multiplicity. Finally, lower values of $\zeta \bar{\beta}$ weaken the agricultural-sector dispersion force working through parameter $d$, which tends to result in multiplicity when trade costs are high. Thus, as shown in Figure 7, lower values of $\zeta$ (stronger input-output loop) push towards multiplicity of equilibria.

\footnotetext{
${ }^{27}$ The tendency for equalization of manufacturing labor income across the regions for small values of $\beta$ and correspondingly large values of $w^{A} / w_{n}$ can be seen from the manufacturing good market clearing condition (6). With higher values of $w^{A} / w_{n}$, the right-hand side of (6) tends to be more driven by the terms $w^{A} \bar{L}_{n}^{A}$, which are the same across the regions in the symmetric case.
} 


\section{Concluding Remarks}

This paper provides a complete characterization of equilibria in a two-region version of the Allen-Arkolakis (AA) economy extended to include an agricultural sector and an input-output loop, for which Krugman (1991) is a special case. Although our analysis yields the full set of equilibria (including whether they are locally stable) for any set of parameters, we direct most of our attention to the role of a few key parameters: $\chi$, which captures the net effect of agglomeration and congestion externalities in manufacturing; $\varepsilon$, which captures the strength of the terms-of-trade dispersion force in manufacturing; $\beta$, which captures the importance of agriculture; and $\left\{\tau_{12}, \tau_{21}\right\}$, which capture trade costs.

Allen and Arkolakis (2014) have already established that the equilibrium is unique if $\chi \leq 0$. We show that this sufficient condition for uniqueness is too strong in the sense that there are conditions under which uniqueness is ensured for positive $\chi$ as long as $\alpha \leq 1$ (or $\chi \leq 1 / \varepsilon$ ). Thus, for example, there are explicit conditions on parameters such that the equilibrium is unique even if agglomeration externalities are stronger than congestion externalities, implying that $\chi>0$. This is possible thanks to two dispersion forces that do not play a role in AA's sufficient condition $\chi \leq 0$. First, a region's terms of trade worsen as it expands, and so a lower trade elasticity or lower trade costs (which increase the relevance of the terms of trade for a region's welfare) make uniqueness more likely. In the extreme, the equilibrium is unique under frictionless trade for all $\alpha<1$, as shown in Proposition 2. The second dispersion force arises thanks to the presence of agriculture, which is captured by $\beta<1$. This lowers the share of manufacturing in consumption, which weakens the link between a region's size, its manufacturing price index, and the welfare of mobile workers, and thus tends to make uniqueness more likely. In the extreme, the equilibrium is unique for all trade costs if $\beta$ is sufficiently low, as shown for the case with no input-output loop in Corollary 2.

These two dispersion forces have different implications for the role of trade costs in affecting whether there is a unique equilibrium. In the special case with no agriculture, for which we give a complete characterization of equilibria in Proposition 5 , for a given ratio of trade costs (i.e., $\tau_{12} / \tau_{21}=c$ for some $c$ ) the equilibrium is unique if and only if trade costs are below a certain threshold. The presence of agriculture complicates this simple relationship between trade costs and uniqueness. Immobile agriculture workers affect the location of manufacturing demand, and this matters for the location of manufacturing production more when trade costs are higher. Thus, whereas higher trade costs

weaken the terms-of-trade dispersion force, they actually strengthen the dispersion force associated with agriculture. This explains why for certain parameter configurations we 
now have a unique equilibrium with low or high trade costs and multiple equilibria for intermediate levels of trade costs.

The modern quantitative spatial economics literature typically uses some version of the AA model with parameter values such that $\chi \leq 0$, ensuring a unique equilibrium. However, theory-consistent estimates by Bartelme (2018) of key parameters of the AA model imply that the assumption $\chi \leq 0$ might not be supported by the data at least at a certain level of aggregation. Moreover, Baum-Snow et al. (2020) argue that a uniqueequilibrium version of the AA model is at odds with their empirical finding that infrastructure improvements in China lead to concentration of economic activity. At the same time, He et al. (2020) find the opposite effects when looking at similar data for China but considering different units of aggregation. Our analysis shows that if $0<\alpha<1$ and $\beta \neq 1$ then reducing transport costs can lead to intermittent regions of concentration and dispersion of economic activity. This could be a potential explanation for the mixed results in the empirical literature.

The main limitation of our analysis, of course, is that we restrict it to two regions. With more than two regions, it is straightforward to establish that AA's sufficient condition for uniqueness, $\chi \leq 0$, is valid even when there is agriculture and an input-output loop, $\beta, \zeta<1$. It should also be possible to generalize to more than two regions our result in Proposition 3 that if $\alpha<1$ then there is a unique equilibrium if trade costs or $\beta$ are low enough. Broadly speaking, we see our results as suggestive of the importance of trade costs, the trade elasticity, and fixed factors (here agriculture) in determining whether the equilibrium is unique in more general environments. Beyond that, providing tight conditions on parameters that ensure that the equilibrium is unique with more than two regions seems very challenging.

\section{References}

Allen, T. and ARKOlAKIS, C. (2014). Trade and the Topography of the Spatial Economy. The Quarterly Journal of Economics, 129 (3), 1085-1140.

ARMington, P. S. (1969). A theory of demand for products distinguished by place of production. Staff Papers (International Monetary Fund), 16 (1), 159-178.

Bartelme, D. (2018). Trade Costs and Economic Geography: Evidence from the US. Mimeo.

Baum-Snow, N., Henderson, J. V., Turner, M. A., Zhang, Q. and Brandt, L. 
(2020). Does investment in national highways help or hurt hinterland city growth? Journal of Urban Economics, 115, 103124, cities in China.

COMbes, P.-P., MAYER, T. and THISSE, J.-F. (2008). Economic Geography: The Integration of Regions and Nations. Princeton University Press.

HE, G., XIE, Y. and ZHANG, B. (2020). Expressways, gdp, and the environment: The case of china. Journal of Development Economics, 145, 102485.

Helpman, E. (1998). The Size of Regions. Topics in public economics: Theoretical and applied analysis, pp. 33-54.

KRUGMAN, P. (1991). Increasing Returns and Economic Geography. Journal of Political Economy, 99 (3), 483-499.

Kucheryavyy, K., Lyn, G. and Rodríguez-Clare, A. (forthcoming). Grounded by Gravity: A Well-Behaved Trade Model with Industry-Level External Economies of Scale. American Economic Journal: Macroecnomics.

REDDING, S. J. (2022). Chapter 3 - Trade and Geography. In G. Gopinath, E. Helpman and K. Rogoff (eds.), Handbook of International Economics: International Trade, Volume 5, Handbook of International Economics, vol. 5, Elsevier, pp. 147-217.

Robert-Nicoud, F. (2005). The Structure of Simple 'New Economic Geography' Models (or, On Identical Twins). Journal of Economic Geography, 5 (2), 201-234.

Sidorov, A. (2011). The Impact of Exogenous Asymmetry on Trade and Agglomeration in CorePeriphery Model. MPRA Paper 32826. 


\section{Appendices}

\section{A Summary of Notation and Definitions}

- $\chi \equiv \frac{\psi-\zeta \delta}{1+\delta}$

- $\alpha \equiv \chi \varepsilon$;

- $\mu \equiv \beta(\chi+\zeta)+(1-\zeta)$;

- $\gamma \equiv \frac{\bar{L}_{1}^{A}}{\bar{L}_{1}^{A}+\bar{L}_{2}^{A}}$;

- $\bar{\gamma} \equiv 1-\gamma$;

- $\bar{\beta} \equiv 1-\beta$;

- $G \equiv\left(\frac{\bar{u}_{1}}{\bar{u}_{2}}\right)^{\alpha+\zeta \varepsilon}\left(\frac{\bar{A}_{1}}{\bar{A}_{2}}\right)^{\varepsilon}$;

- $x \equiv\left(\frac{w_{1}^{\zeta} P_{1}^{1-\zeta} /\left(\bar{A}_{1} L_{1}^{\psi}\right)}{w_{2}^{\zeta} P_{2}^{1-\zeta} /\left(\bar{A}_{2} L_{2}^{\psi}\right)}\right)^{\mathcal{E}}$;

- $g_{\phi}(x) \equiv\left(1+\phi_{1} x\right) /\left(\phi_{2}+x\right)$;

- $g_{d}(x) \equiv\left(1+d_{1} x\right) /\left(d_{2}+x\right)$;

- $d_{1} \equiv \zeta \gamma \bar{\beta} \phi_{2}^{-1}+(1-\zeta \gamma \bar{\beta}) \phi_{1}$;

- $d_{2} \equiv \zeta \bar{\gamma} \bar{\beta} \phi_{1}^{-1}+(1-\zeta \bar{\gamma} \bar{\beta}) \phi_{2}$;

- $\bar{\mu}_{a} \equiv \frac{1+\sqrt{\phi_{1} \phi_{2}}}{1-\sqrt{\phi_{1} \phi_{2}}} \cdot \frac{1-2 \alpha+\sqrt{d_{1} d_{2}}}{1+\sqrt{d_{1} d_{2}}}$;

- $\bar{\mu}_{b} \equiv \alpha \cdot \frac{\left(d_{1} d_{2}-1\right) \phi_{1} \phi_{2}}{1-\phi_{1} \phi_{2}}+(1-\alpha) \cdot \frac{1+\sqrt{\phi_{1} \phi_{2}}}{1-\sqrt{\phi_{1} \phi_{2}}} ;$

- $\overline{\bar{\mu}} \equiv \frac{d_{1} d_{2}-1}{\left(1-\phi_{1} \phi_{2}\right) d_{1} d_{2}} \cdot \frac{1-2 \alpha+\sqrt{d_{1} d_{2}}}{\sqrt{d_{1} d_{2}}-1}$

- $\tilde{\phi}_{1} \equiv \frac{1-\mu}{1+\mu} \cdot \frac{\zeta \bar{\gamma} \bar{\beta}}{1-\zeta \bar{\gamma} \bar{\beta}}\left(\frac{\gamma}{\bar{\gamma}}\right)^{\frac{1}{1-\mu}} G^{\frac{1}{1-\mu}}$;

- $\tilde{\phi}_{2} \equiv \frac{1-\mu}{1+\mu} \cdot \frac{\zeta \gamma \bar{\beta}}{1-\zeta \gamma \bar{\beta}}\left(\frac{\gamma}{\bar{\gamma}}\right)^{-\frac{1}{1-\mu}} G^{-\frac{1}{1-\mu}}$; 
- $\eta \equiv \frac{\mu+2 \alpha-1}{\mu+1}$

- In the case when $\eta \geq \sqrt{\phi_{1} \phi_{2}}$ (equivalent to $\mu \leq \bar{\mu}_{a}$ for $\beta=1$ ),

$$
\begin{aligned}
& \Gamma_{1} \equiv \Xi \cdot\left[\phi_{1} \phi_{2}^{\mu}\right]^{-1}\left(\eta-\phi_{1} \phi_{2}-D\right)^{1-\alpha}\left(\eta+\phi_{1} \phi_{2}+D\right)^{\mu+\alpha}, \\
& \Gamma_{2} \equiv \Xi \cdot\left[\phi_{1} \phi_{2}^{\mu}\right]^{-1}\left(\eta-\phi_{1} \phi_{2}+D\right)^{1-\alpha}\left(\eta+\phi_{1} \phi_{2}-D\right)^{\mu+\alpha}
\end{aligned}
$$

with $D \equiv \sqrt{\left(1-\phi_{1} \phi_{2}\right)\left(\eta^{2}-\phi_{1} \phi_{2}\right)}$ and

$$
\Xi \equiv \frac{G \cdot(\mu+1)^{\mu+1}}{2^{\mu+1}(1-\alpha)^{1-\alpha}(\mu+\alpha)^{\mu+\alpha}}
$$

\section{B Proofs for Section 3}

\section{B.1 Proof of Proposition 1}

In equilibrium, at least one region is inhabited. Hence, $L_{i}^{\varepsilon \psi}\left(w_{i}^{\zeta} P_{i}^{1-\zeta}\right)^{-\varepsilon}>0$ for at least one $i$ - otherwise $w_{i}^{\zeta} P_{i}^{1-\zeta}$ needs to be infinite for all $i$ with $L_{i}>0$, which cannot be an equilibrium. Moreover, if $L_{i}^{\varepsilon \psi}\left(w_{i}^{\zeta} P_{i}^{1-\zeta}\right)^{-\varepsilon}$ is not a finite number for some $i$, then expression (4) for $P_{n}$ implies that $P_{n}=0$ for all $n$, which cannot be an equilibrium either. Hence, $L_{i}^{\varepsilon \psi}\left(w_{i}^{\zeta} P_{i}^{1-\zeta}\right)^{-\varepsilon}$ is finite for all $i$ and positive for the inhabited regions. Expression (4) for $P_{n}$ then implies that $P_{n}$ is positive and finite for all $n$ (regardless of whether the region is inhabited or not).

The rest of the proof of Proposition 1 closely follows the AA's proof of existence of irregular equilibria. Namely, we consider the equilibrium conditions with $L_{i}>0$ for all $i$ and take the limit as $L_{i} \rightarrow 0$ for one of the regions $i=1$ or $i=2$.

The manufactured goods market clearing condition (6) implies

$$
w_{i}=\bar{A}_{i}^{\frac{\varepsilon}{1+\zeta \varepsilon}} L_{i}^{\frac{\varepsilon \psi-1}{1+\zeta \varepsilon}} P_{i}^{-\frac{(1-\zeta) \varepsilon}{1+\zeta \varepsilon}}\left[\sum_{n=1}^{N} \tau_{n i}^{-\varepsilon} P_{n}^{\varepsilon}\left[\zeta \beta\left(w_{n} L_{n}+w^{A} \bar{L}_{n}^{A}\right)+(1-\zeta) w_{n} L_{n}\right]\right]^{\frac{1}{1+\zeta \varepsilon}}
$$


Substituting this into (2) and combining with (3), we get

$$
\begin{aligned}
U_{i}= & \bar{u}_{i} \bar{A}_{i}^{\frac{\varepsilon}{1+\zeta \varepsilon}}\left[w^{A}\right]^{-(1-\beta)} L_{i}^{\frac{(1+\delta)(\alpha-1)}{1+\zeta \varepsilon}} P_{i}^{-\beta-\frac{(1-\zeta) \varepsilon}{1+\zeta \varepsilon}} \\
& \times\left[\sum_{n=1}^{N} \tau_{n i}^{-\varepsilon} P_{n}^{\varepsilon}\left[\zeta \beta\left(w_{n} L_{n}+w^{A} \bar{L}_{n}^{A}\right)+(1-\zeta) w_{n} L_{n}\right]\right]^{\frac{1}{1+\zeta \varepsilon}}
\end{aligned}
$$

Taking the limit $L_{i} \rightarrow 0$, we see that $U_{i} \rightarrow \infty$ if $\alpha<1$ (as we have argued above, $P_{i}$ has to be finite for all regions, including regions with $L_{i}=0$ ). Hence, in the case with $\alpha<1$ irregular equilibria are not possible. This proves part (i) of Proposition 1.

Next, consider the case with $\alpha>1$ and suppose that $L_{1}=\bar{L}$ and $L_{2}=0$. Then (16) implies that $U_{1}$ is a finite positive number while $U_{2}=0$, and thus condition (8) is satisfied with $\bar{U}=U_{1}$. Therefore, the allocation $L_{1}=\bar{L}$ and $L_{2}=0$ is an equilibrium. Clearly, the mirror image allocation $L_{1}=0$ and $L_{2}=\bar{L}$ is also an equilibrium. This proves part (iii) of Proposition 1.

Finally, consider the case with $\alpha=1$. Consider the allocation $L_{1}=\bar{L}$ and $L_{2}=0$. We have $P_{1}=w_{1} /\left(A_{1} \bar{L}^{\psi}\right)^{\frac{1}{\zeta}}$ and $P_{2}=\tau_{21} P_{1}$. Also, $\lambda_{11}=\lambda_{21}=1$ and $\lambda_{12}=\lambda_{22}=0$. From (16) we find

$$
\begin{aligned}
U_{1}= & \bar{u}_{1} \bar{A}_{1}^{\frac{\beta}{\zeta}}\left[w^{A}\right]^{-(1-\beta)} w_{1}^{\frac{\zeta \varepsilon}{1+\zeta \varepsilon}-\beta} \bar{L}^{-\frac{\psi}{\zeta}\left(\frac{\zeta \varepsilon}{1+\zeta \varepsilon}-\beta\right)} \\
\times & {\left[(\zeta \beta+1-\zeta) w_{1} \bar{L}+\zeta \beta w^{A} \bar{L}_{1}^{A}+\zeta \beta w^{A} \bar{L}_{2}^{A}\right]^{\frac{1}{1+\zeta \varepsilon}}, } \\
U_{2}= & \bar{u}_{2} \bar{A}_{1}^{\frac{\beta}{\zeta}-\frac{\varepsilon}{1+\zeta \varepsilon}} \bar{A}_{2}^{\frac{\varepsilon}{1+\zeta \varepsilon}}\left[w^{A}\right]^{-(1-\beta)} \tau_{21}^{-\beta-\frac{(1-\zeta) \varepsilon}{1+\zeta \varepsilon}} w_{1}^{\frac{\zeta \varepsilon}{1+\zeta \varepsilon}-\beta} \bar{L}^{-\frac{\psi}{\zeta}\left(\frac{\zeta \varepsilon}{1+\zeta \varepsilon}-\beta\right)} \\
\times & {\left[\tau_{12}^{-\varepsilon}\left[(\zeta \beta+1-\zeta) w_{1} \bar{L}+\zeta \beta w^{A} \bar{L}_{1}^{A}\right]+\tau_{21}^{\varepsilon} \zeta \beta w^{A} \bar{L}_{2}^{A}\right]^{\frac{1}{1+\zeta \varepsilon}} . }
\end{aligned}
$$

The equilibrium condition that $U_{1} \geq U_{2}$ gives

$$
\begin{aligned}
& (\zeta \beta+1-\zeta) w_{1} \bar{L}+\zeta \beta w^{A} \bar{L}_{1}^{A}+\zeta \beta w^{A} \bar{L}_{2}^{A} \\
& \quad \geq\left[\phi_{1}\left[(\zeta \beta+1-\zeta) w_{1} \bar{L}+\zeta \beta w^{A} \bar{L}_{1}^{A}\right]+\phi_{2}^{-1} \zeta \beta w^{A} \bar{L}_{2}^{A}\right] G^{-1} \phi_{2}^{\mu}
\end{aligned}
$$

where $G \equiv\left(\bar{u}_{1} / \bar{u}_{2}\right)^{1+\varepsilon}\left(\bar{A}_{1} / \bar{A}_{2}\right)^{\varepsilon}, \mu \equiv \beta(1 / \varepsilon+\zeta)+(1-\zeta)$, and $\phi_{1} \equiv \tau_{12}^{-\varepsilon}$ and $\phi_{2} \equiv \tau_{21}^{-\varepsilon}$.

Consider the case with $\beta=1$ and $\bar{L}_{1}^{A}=\bar{L}_{2}^{A}=0$ (no agricultural sector in both regions). In this case, inequality (17) becomes $G \geq \phi_{1} \phi_{2}^{\mu}$. Thus, we get that the allocation $L_{1}=\bar{L}$ and $L_{2}=0$ is an equilibrium if and only if $G \geq \phi_{1} \phi_{2}^{\mu}$, which is the condition in part (ii.a) of Proposition 1 for $\beta=1$ (or, equivalently, for $\bar{\beta}=0$ ). 
Now consider the case with $0<\beta<1$. In this case, the agricultural goods market clearing condition (7) gives

$$
\frac{w_{1}}{w^{A}}=\frac{\beta}{1-\beta} \cdot \frac{\bar{L}_{1}^{A}+\bar{L}_{2}^{A}}{\bar{L}} .
$$

Substituting this into (17) and after doing some algebra, we get the condition from part (ii.a) of Proposition 1

$$
G \geq \zeta \bar{\gamma} \bar{\beta} \phi_{2}^{\mu-1}+\phi_{1} \phi_{2}^{\mu}(1-\zeta \bar{\gamma} \bar{\beta})
$$

Proving part (ii.b) of Proposition 1 can be done in an analogous manner.

\section{B.2 Derivation of the Key Equation for Regular Equilibria Analysis}

Denote $l \equiv L_{2} / L_{1}, p \equiv P_{2} / P_{1}$, and $w \equiv w_{2} / w_{1}$. Also, denote $a \equiv\left(\bar{u}_{2} / \bar{u}_{1}\right)^{\varepsilon}, b \equiv\left(\bar{A}_{2} / \bar{A}_{1}\right)^{\varepsilon}$, and $\phi_{1} \equiv \tau_{12}^{-\varepsilon}$ and $\phi_{2} \equiv \tau_{21}^{-\varepsilon}$. In the case of regular equilibria, the complementary slackness condition (8) implies that both regions have the same welfare $\bar{U}$, and we can combine (2)(3) for the two regions and get

$$
1=\frac{U_{1}}{U_{2}}=a^{-\frac{1}{\varepsilon}} w^{-1} l^{\delta} p^{\beta}
$$

Dividing onto each other price indices for the two regions given by expression (4), we get

$$
p=\left[\frac{\phi_{2}+b l^{\varepsilon \psi} w^{-\zeta \varepsilon} p^{-(1-\zeta) \varepsilon}}{1+\phi_{1} b l^{\varepsilon \psi} w^{-\zeta \varepsilon} p^{-(1-\zeta) \varepsilon}}\right]^{-\frac{1}{\varepsilon}} .
$$

After substituting this expression into (18) and doing some algebra, we get

$$
1=\left(\frac{U_{1}}{U_{2}}\right)^{\frac{\varepsilon}{\beta}}=a^{-\frac{1}{\beta}} w^{-\frac{\varepsilon}{\beta}} l^{\frac{\varepsilon \delta}{\beta}} \frac{1+\phi_{1} b l^{\varepsilon \psi} w^{-\zeta \varepsilon} p^{-(1-\zeta) \varepsilon}}{\phi_{2}+b l^{\varepsilon \psi} w^{-\zeta \varepsilon} p^{-(1-\zeta) \varepsilon}} .
$$

Next, the manufactured and agricultural goods market clearing conditions (6) and (7) 
for the first region can be written as

$$
\begin{aligned}
& 1=\frac{1}{1+\phi_{1} b l^{\varepsilon \psi} w^{-\zeta \varepsilon} p^{-(1-\zeta) \varepsilon}}\left[\zeta \beta\left(1+\frac{w^{A} \bar{L}_{1}^{A}}{w_{1} L_{1}}\right)+(1-\zeta)\right] \\
& +\frac{\phi_{2}}{\phi_{2}+b l^{\varepsilon \psi} w^{-\zeta \varepsilon} p^{-(1-\zeta) \varepsilon}}\left[\zeta \beta\left(w l+\frac{w^{A} \bar{L}_{2}^{A}}{w_{1} L_{1}}\right)+(1-\zeta) w l\right], \\
& \beta\left(\frac{w^{A} \bar{L}_{1}^{A}+w^{A} \bar{L}_{2}^{A}}{w_{1} L_{1}}\right)=(1-\beta)(1+w l) .
\end{aligned}
$$

Solving for $w_{1} L_{1}$ from the second equation and substituting into the first, gives

$$
\begin{aligned}
& 1=\frac{1}{1+\phi_{1} b l^{\varepsilon \psi} w^{-\zeta \varepsilon} p^{-(1-\zeta) \varepsilon}}(1-\zeta \bar{\gamma} \bar{\beta}+\zeta \gamma \bar{\beta} w l) \\
& \quad+\frac{\phi_{2}}{\phi_{2}+b l^{\varepsilon \psi} w^{-\zeta \varepsilon} p^{-(1-\zeta) \varepsilon}}(\zeta \bar{\gamma} \bar{\beta}+(1-\zeta \gamma \bar{\beta}) w l)
\end{aligned}
$$

where $\bar{\beta} \equiv 1-\beta, \gamma \equiv \bar{L}_{1}^{A} /\left(\bar{L}_{1}^{A}+\bar{L}_{2}^{A}\right)$, and $\bar{\gamma} \equiv 1-\gamma$.

The equilibrium system of equations is given by (19)-(21). Let us introduce the change of variables $x_{1} \equiv b l^{\varepsilon \psi} w^{-\zeta \varepsilon} p^{-(1-\zeta) \varepsilon}$ and $x_{2} \equiv l w$. Then $l=b^{-\frac{1}{\varepsilon(\psi+\zeta)}} x_{1}^{\frac{1}{\varepsilon(\psi+\zeta)}} x_{2}^{\frac{\zeta}{\psi+\zeta}} p^{\frac{1-\zeta}{\psi+\zeta}}$ and $w=b^{\frac{1}{\varepsilon(\psi+\zeta)}} x_{1}^{-\frac{1}{\varepsilon(\psi+\zeta)}} x_{2}^{\frac{\psi}{\psi+\zeta}} p^{-\frac{1-\zeta}{\psi+\zeta}}$, and equations (20) and (21) become

$$
\begin{aligned}
& p=\left(\frac{\phi_{2}+x_{1}}{1+\phi_{1} x_{1}}\right)^{-\frac{1}{\varepsilon}}, \\
& 1=\left(\frac{U_{1}}{U_{2}}\right)^{\frac{\varepsilon}{\beta}}=a^{-\frac{1}{\beta}} b^{-\frac{1+\delta}{\beta(\psi+\zeta)}} x_{1}^{\frac{1+\delta}{\beta(\psi+\zeta)}} x_{2}^{-\frac{\varepsilon(\psi-\zeta \delta)}{\beta(\psi+\zeta)}} p^{\frac{\varepsilon(1-\zeta)(1+\delta)}{\beta(\psi+\zeta)}} \frac{1+\phi_{1} x_{1}}{\phi_{2}+x_{1}}, \\
& x_{2}=\frac{\left[\zeta \bar{\gamma} \bar{\beta}+(1-\zeta \bar{\gamma} \bar{\beta}) \phi_{1} \phi_{2}+\phi_{1} x_{1}\right] x_{1}}{\phi_{2}+\left(\zeta \gamma \bar{\beta}+(1-\zeta \gamma \bar{\beta}) \phi_{1} \phi_{2}\right) x_{1}} .
\end{aligned}
$$

Substituting $p$ and $x_{2}$ from the first and third equations into the second equation, and after doing some algebra, we get

$$
1=\left(\frac{U_{1}}{U_{2}}\right)^{\frac{(\psi+\zeta) \varepsilon}{1+\delta}}=a^{-\frac{\psi+\zeta}{1+\delta}} b^{-1}\left(\frac{\phi_{1}}{\phi_{2}}\right)^{-\alpha} x_{1}^{1-\alpha}\left(\frac{1+\phi_{1} x_{1}}{\phi_{2}+x_{1}}\right)^{\mu}\left(\frac{1+d_{1} x_{1}}{d_{2}+x_{1}}\right)^{\alpha}
$$

where $\alpha \equiv \frac{(\psi-\zeta \delta) \varepsilon}{1+\delta}, \mu \equiv \frac{\beta(\psi+\zeta)}{1+\delta}+(1-\zeta)=\beta\left(\frac{\alpha}{\varepsilon}+\zeta\right)+(1-\zeta)$, and

$$
d_{1} \equiv \frac{\zeta \gamma \bar{\beta}+(1-\zeta \gamma \bar{\beta}) \phi_{1} \phi_{2}}{\phi_{2}} \quad \text { and } \quad d_{2} \equiv \frac{\zeta \bar{\gamma} \bar{\beta}+(1-\zeta \bar{\gamma} \bar{\beta}) \phi_{1} \phi_{2}}{\phi_{1}} .
$$


In the main text and elsewhere in the appendix we use $x$ instead of $x_{1}$.

\section{B.3 Maximum Number of Regular Equilibria Under Costly Trade}

Proposition 6 (Maximum number of regular equilibria under costly trade). Assume that trade is costly $\left(\phi_{1} \phi_{2}<1\right)$.

(i) If $\alpha \neq 1$ then the economy has at most five regular equilibria.

(ii) If $\alpha=1$ then the economy has at most three regular equilibria.

Proof. Taking the first derivative of $V(x)$ defined in (12), we get $V^{\prime}(x)=x^{-1} W_{2}(x) / W_{1}(x)$, where

$$
\begin{aligned}
& W_{1}(x) \equiv\left(1+\phi_{1} x\right)\left(\phi_{2}+x\right)\left(1+d_{1} x\right)\left(d_{2}+x\right) \\
& W_{2}(x) \equiv(1-\alpha) W_{1}(x)-\mu\left(1-\phi_{1} \phi_{2}\right)\left(1+d_{1} x\right)\left(d_{2}+x\right) x \\
& -\alpha\left(1-d_{1} d_{2}\right)\left(1+\phi_{1} x\right)\left(\phi_{2}+x\right) x .
\end{aligned}
$$

We see that $V^{\prime}(x)=0$ if and only if $W_{2}(x)=0$. Expanding the terms of $W_{2}(x)$, we can see that in the case with $\alpha \neq 1$ it is a 4th degree polynomial, while in the case with $\alpha=1$ function $x^{-1} W_{2}(x)$ is a quadratic polynomial. Since a $n$th degree polynomial has at most $n$ real roots, function $V(x)$ has at most 4 extrema if $\alpha \neq 1$, and it has at most 2 extrema if $\alpha=1$. Since a continuous function with $n$ extrema can intersect the horizontal axis at most $n+1$ times, we get that equation $V(x)=0$ can have at most 5 solutions if $\alpha \neq 1$ and it can have at most 3 solutions if $\alpha=1$.

The fact that for $\alpha \neq 1$ solving equation $V^{\prime}(x)=0$ for $x>0$ is equivalent to finding roots of a fourth-degree polynomial has one more important implication for our analysis. Suppose that $0<x_{1}^{*}<\cdots<x_{M}^{*}$ with $0 \leq M \leq 4$ are distinct positive real solutions to $V^{\prime}(x)=0$. Since roots of a fourth-degree polynomial can be found analytically, we can obtain closed-form (although, very complicated) expressions for $x_{1}^{*}, \ldots, x_{M}^{*}$. These expressions depend only on the exogenous parameters of the model. Each of the points $x_{1}^{*}, \ldots, x_{M}^{*}$ can be either a local extremum of $V(\cdot)$ or an inflection point. Checking the signs of $V\left(x_{1}^{*}\right), \ldots, V\left(x_{M}^{*}\right)$ as well as $\lim _{x \rightarrow 0} V(x)$ and $\lim _{x \rightarrow \infty} V(\infty)$, we can unambiguously determine how many times function $V(\cdot)$ intersects the horizontal axis, which would give us the number of regular equilibria in the original economy. ${ }^{28}$ For example, if $M=0$ then function $V(\cdot)$ is monotonic and so there could be only one regular

\footnotetext{
${ }^{28}$ Our exhaustive analysis of regular equilibria in the Krugman case - provided in Section 2 of the Online Appendix - is based on this logic. The same logic is also used in our analysis of multiplicity of regular equilibria for the AA economy $(\beta=1)$ in the case of $0<\alpha<1$, which is presented in Proposition 5 in the main text.
} 
equilibrium. As another example, suppose that $M=2$ and $V\left(x_{1}^{*}\right)<0, V\left(x_{2}^{*}\right)>0$, $\lim _{x \rightarrow 0} V(x)=\infty$ and $\lim _{x \rightarrow \infty} V(x)=-\infty$. In this case $x_{1}^{*}$ is a local minimum of $V(\cdot)$, $x_{2}^{*}$ is a local maximum of $V(\cdot)$, and $V(\cdot)$ necessarily intersects the horizontal axis three times, implying that there exist three regular equilibria.

\section{B.4 Applying Function $V$ for Stability Analysis}

In this appendix section we show that it is enough to analyze the sign of the derivative of $V$ in order to determine stability of regular equilibria of the economy. Formally, we show that $d \omega / d L_{1}<0$ if and only if $d V(x) / d \ln x>0$. We then use function $V$ to provide results on stability of unique equilibria in the cases with $\alpha \in(0,1)$ and $\alpha>1$.

Here we use the definitions introduced in Appendix B.2. The key result is the following lemma.

Lemma 1. $x$ is a decreasing function of $L_{1}, d \ln x / d \ln L_{1}<0$.

Proof. Note that

$$
l \equiv \frac{L_{2}}{L_{1}}=\frac{\bar{L}-L_{1}}{L_{1}}
$$

is a decreasing function of $L_{1}$. Thus, showing that $d \ln x / d \ln L_{1}<0$ is equivalent to showing that $d \ln x / d \ln l>0$, which, in its turn, is equivalent to showing that $d \ln l / d \ln x>0$, which we do below.

Derivations in Appendix B.2 give

$$
\begin{aligned}
l & =b^{-\frac{1}{\varepsilon(\psi+\zeta)}} x_{1}^{\frac{1}{\varepsilon(\psi+\zeta)}} x_{2}^{\frac{\zeta}{\psi+\zeta}} p^{\frac{1-\zeta}{\psi+\zeta}} \\
& =\left[b^{-1}\left(\frac{\phi_{1}}{\phi_{2}}\right)^{\zeta \varepsilon} x^{1+\zeta \varepsilon}\left[g_{\phi}(x)\right]^{1-\zeta} \cdot\left[g_{d}(x)\right]^{-\zeta \varepsilon}\right]^{\frac{1}{\varepsilon(\psi+\zeta)}},
\end{aligned}
$$

where in the second line we used $x$ instead of $x_{1}$ (as we do everywhere in the paper). We have

$$
\varepsilon \cdot(\psi+\zeta) \frac{d \ln l}{d \ln x}=(1+\zeta \varepsilon)-(1-\zeta)\left(1-\phi_{1} \phi_{2}\right) f_{\phi}(x)+\zeta \varepsilon \cdot\left(1-d_{1} d_{2}\right) f_{d}(x),
$$

where

$$
f_{\phi}(x) \equiv \frac{x}{\left(1+\phi_{1} x\right)\left(\phi_{2}+x\right)} \quad \text { and } \quad f_{d}(y) \equiv \frac{x}{\left(1+d_{1} x\right)\left(d_{2}+x\right)} .
$$

Function $f_{\phi}(x)$ is a concave function that achieves its maximum at $x_{\phi}^{*}=\sqrt{\phi_{1}^{-1} \phi_{2}}$. Similarly, $f_{d}(x)$ is a concave function that achieves its maximum at $x_{d}^{*}=\sqrt{d_{1}^{-1} d_{2}}$. Thus, we 
have that for all $x>0$,

$$
0<f_{\phi}(x) \leq f_{\phi}\left(x_{\phi}^{*}\right)=\frac{1}{\left(1+\sqrt{\phi_{1} \phi_{2}}\right)^{2}} \quad \text { and } \quad 0<f_{d}(x) \leq f_{d}\left(x_{d}^{*}\right)=\frac{1}{\left(1+\sqrt{d_{1} d_{2}}\right)^{2}} .
$$

This implies that

$$
(1-\zeta)\left(1-\phi_{1} \phi_{2}\right) f_{\phi}(x) \leq \frac{(1-\zeta)\left(1-\sqrt{\phi_{1} \phi_{2}}\right)}{1+\sqrt{\phi_{1} \phi_{2}}}<1,
$$

and so

$$
\varepsilon \cdot(\psi+\zeta) \frac{d \ln l}{d \ln x}>\zeta \varepsilon \cdot\left[1+\left(1-d_{1} d_{2}\right) f_{d}(x)\right] .
$$

Next, if $d_{1} d_{2} \leq 1$ then we immediately get $d \ln l / d \ln x>0$. If $d_{1} d_{2}>1$ then the bound (22) on $f_{d}(x)$ implies

$$
\left(d_{1} d_{2}-1\right) f_{d}(x) \leq \frac{\sqrt{d_{1} d_{2}}-1}{\sqrt{d_{1} d_{2}}+1}<1,
$$

and so $1+\left(1-d_{1} d_{2}\right) f_{d}(x)>0$, which implies that $d \ln l / d \ln x>0$.

The derivations in Appendix B.2 show that $V(x)=\frac{(\psi+\zeta) \varepsilon}{1+\delta} \ln \omega$, where $\omega \equiv U_{1} / U_{2}$. Then we can write

$$
\frac{d \ln \omega}{d \ln L_{1}}=\frac{1+\delta}{(\psi+\zeta) \varepsilon} \cdot \frac{d V(x)}{d \ln x} \cdot \frac{d \ln x}{d \ln L_{1}} .
$$

We have $\frac{1+\delta}{(\psi+\zeta) \varepsilon}>0$ and also, according to Lemma 1 , we have $d \ln x / d L_{1}<0$. Therefore, $d \ln \omega / d \ln L_{1}<0$ if and only if $d V(x) / d \ln x>0$. Obviously, for regular equilibria the sign of $d \ln \omega / d \ln L_{1}$ is the same as the sign of $d \omega / d L_{1}$. Thus, we get that $d \omega / d L_{1}<0$ if and only if $d V(x) / d \ln x>0$.

We now use function $V$ to prove results on stability of unique equilibria in the cases with $\alpha \in(0,1)$ and $\alpha>1$.

Claim 1. For $\alpha \in(0,1)$, if an equilibrium is unique then it is generically locally stable. For $\alpha>1$, if an equilibrium is unique then it is generically unstable.

Proof. Observe that if $0<\alpha<1$ then $\lim _{x \rightarrow 0} V(x)=-\infty$ and $\lim _{x \rightarrow \infty} V(x)=\infty$. Therefore, if there is only one $\tilde{x}>0$ such that $V(\tilde{x})=0$, it has to be the case that function $V(x)$ cuts the horizontal axis from below, implying that $V^{\prime}(\tilde{x}) \geq 0$. Generically, $V^{\prime}(\tilde{x}) \neq 0$ and so the corresponding equilibrium is generically locally stable.

Similarly, for $\alpha>1$ we have $\lim _{x \rightarrow 0} V(x)=\infty$ and $\lim _{x \rightarrow \infty} V(x)=-\infty$. And so if there is only one $\tilde{x}>0$ such that $V(\tilde{x})=0$, it has to be the case that function $V(x)$ 
cuts the horizontal axis from above. Thus, the corresponding equilibrium is generically unstable.

\section{B.5 Proof of Proposition 3}

In the proof of Proposition 3, we are going to use the following result. ${ }^{29}$

Lemma 2. If $\phi_{1} \phi_{2}<1$, then there exist $\bar{\epsilon}_{1}, \bar{\epsilon}_{2} \in(0,1)$ such that for for any $\epsilon_{1} \in\left[0, \bar{\epsilon}_{1}\right]$ and $\epsilon_{2} \in\left[0, \bar{\epsilon}_{2}\right]$ and any $y$ we have

$$
\left(1-\epsilon_{1}\right)^{-1}\left(\phi_{1} \phi_{2}\right) f_{\phi}(x) \leq f_{d}(x) \leq\left(1-\epsilon_{2}\right)\left(d_{1} d_{2}\right)^{-1} f_{\phi}(x), \text { for all } x>0,
$$

where

$$
f_{\phi}(x) \equiv \frac{x}{\left(1+\phi_{1} x\right)\left(\phi_{2}+x\right)} \quad \text { and } \quad f_{d}(y) \equiv \frac{x}{\left(1+d_{1} x\right)\left(d_{2}+x\right)} .
$$

Proof. Given the definitions of $d_{1}$ and $d_{2}$

$$
d_{1} \equiv \frac{\zeta \gamma \bar{\beta}+(1-\zeta \gamma \bar{\beta}) \phi_{1} \phi_{2}}{\phi_{2}} \quad \text { and } \quad d_{2} \equiv \frac{\zeta \bar{\gamma} \bar{\beta}+(1-\zeta \bar{\gamma} \bar{\beta}) \phi_{1} \phi_{2}}{\phi_{1}}
$$

we can write $d_{1}=u_{1} \phi_{2}^{-1}$ and $d_{2}=u_{2} \phi_{1}^{-1}$, where

$$
u_{1} \equiv \zeta \gamma \bar{\beta}+(1-\zeta \gamma \bar{\beta}) \phi_{1} \phi_{2} \quad \text { and } \quad u_{2} \equiv \zeta \bar{\gamma} \bar{\beta}+(1-\zeta \bar{\gamma} \bar{\beta}) \phi_{1} \phi_{2}
$$

Then the definitions of $f_{\phi}(x)$ and $f_{d}(x)$ imply

$$
f_{\phi}(x)=\left[\phi_{1} \phi_{2}\right]^{-1} h_{d}(x) f_{d}(x) \text { and } f_{d}(x)=\left[d_{1} d_{2}\right]^{-1} h_{\phi}(x) f_{\phi}(x),
$$

where

$$
h_{\phi}(x) \equiv \frac{1+\phi_{1} x}{1+u_{2}^{-1} \phi_{1} x} \cdot \frac{\phi_{2}+x}{u_{1}^{-1} \phi_{2}+x} \quad \text { and } \quad h_{d}(x) \equiv \frac{1+d_{1} x}{1+u_{1}^{-1} d_{1} x} \cdot \frac{d_{2}+x}{u_{2}^{-1} d_{2}+x} .
$$

Clearly, $u_{1}<1$ and $u_{2}<1$ as long as $\phi_{1} \phi_{2}<1$. This implies that $h_{\phi}(x)<1$ and $h_{d}(x)<1$ for all $x>0$. Let us focus on $h_{\phi}(x)$ and show that a stronger result holds: there exists some $0<\epsilon_{\phi}<1$ such that $h_{\phi}(x) \leq 1-\epsilon_{\phi}$. Proving a similar result for $h_{d}(x)$ can be done in an analogous manner.

\footnotetext{
${ }^{29}$ Note that Lemma 2 does not involve parameters $\alpha$ and $\mu$.
} 
Consider the following function

$$
\tilde{h}_{\phi}(x) \equiv \frac{u_{1}^{-1} \phi_{2}+x}{\phi_{2}+x}-\frac{1+\phi_{1} x}{1+u_{2}^{-1} \phi_{1} x} .
$$

We have

$$
\tilde{h}_{\phi}^{\prime}(x)=\frac{\left(u_{2}^{-1}-1\right) \phi_{1} \cdot\left[\phi_{2}-B+\left(1-u_{2}^{-1} \phi_{1} B\right) x\right] \cdot\left[\phi_{2}+B+\left(1+u_{2}^{-1} \phi_{1} B\right) x\right]}{\left(1+u_{2}^{-1} \phi_{1} x\right)^{2}\left(\phi_{2}+x\right)^{2}},
$$

where

$$
B \equiv \sqrt{\frac{\left(u_{1}^{-1}-1\right) \phi_{2}}{\left(u_{2}^{-1}-1\right) \phi_{1}}} .
$$

Let us go through different cases for the signs of $\left(\phi_{2}-B\right)$ and $\left(1-u_{2}^{-1} \phi_{1} B\right)$.

(i) $\phi_{2}-B=0$ and $1-u_{2}^{-1} \phi_{1} B=0$. Then $\tilde{h}_{\phi}(x)=1-u_{2}>0$ for all $x>0$.

(ii) $\phi_{2}-B<0$ and $1-u_{2}^{-1} \phi_{1} B \leq 0$; or $\phi_{2}-B=0$ and $1-u_{2}^{-1} \phi_{1} B<0$. In these cases $\tilde{h}_{\phi}^{\prime}(x)<0$ for all $x>0$ and, thus, $\tilde{h}_{\phi}(x)$ is a decreasing function and $\tilde{h}_{\phi}(x) \geq$ $\lim _{x \rightarrow 0} \tilde{h}_{\phi}(x)=1-u_{2}>0$ for all $x>0$.

(iii) $\phi_{2}-B>0$ and $1-u_{2}^{-1} \phi_{1} B \geq 0$; or $\phi_{2}-B=0$ and $1-u_{2}^{-1} \phi_{1} B>0$. In these cases $\tilde{h}_{\phi}^{\prime}(x)>0$ and, thus, $\tilde{h}_{\phi}(x)$ is an increasing function and $\tilde{h}_{\phi}(x) \geq \lim _{x \rightarrow 0} \tilde{h}_{\phi}(x)=$ $u_{1}^{-1}-1>0$ for all $x>0$.

(iv) $\phi_{2}-B>0$ and $1-u_{2}^{-1} \phi_{1} B<0$. In this case $\tilde{h}_{\phi}(x)$ is a concave function bounded below by the minimum of $\lim _{x \rightarrow 0} \tilde{h}_{\phi}(x)$ and $\lim _{x \rightarrow \infty} \tilde{h}_{\phi}(x)$, which are both positive.

(v) $\phi_{2}-B<0$ and $1-u_{2}^{-1} \phi_{1} B>0$. This is the most involved case, in which $\tilde{h}_{\phi}(x)$ is a convex function that achieves its global minimum at $x^{*}=\frac{B-\phi_{2}}{1-u_{2}^{-1} \phi_{1} B}$. We have

$$
\tilde{h}_{\phi}\left(x^{*}\right)=\frac{u_{1}^{-1} \phi_{2}\left(1-u_{2}^{-1} \phi_{1} B\right)+\left(u_{2}^{-1}-1\right) \phi_{1} B^{2}+\left(\phi_{1} B-1\right) \phi_{2}}{\left(1-u_{2}^{-1} \phi_{1} \phi_{2}\right) B} .
$$

Substituting $B^{2}$ from (25) into this expression and after doing some algebra, we get

$$
\tilde{h}_{\phi}\left(x^{*}\right)=\frac{\left[2\left(1-u_{1}\right) u_{2}-\left(1-u_{1} u_{2}\right) \phi_{1} B\right] \phi_{2}}{\left(1-u_{2}^{-1} \phi_{1} \phi_{2}\right) u_{1} u_{2} B} .
$$

Given our case-(v) assumptions that $\phi_{2}<B$ and $1-u_{2}^{-1} \phi_{1} B>0$, we get $1-u_{2}^{-1} \phi_{1} \phi_{2}>$ $1-u_{2}^{-1} \phi_{1} B>0$. Thus, the denominator of (26) is positive. 
Next, suppose that $1-2 u_{1}+u_{1} u_{2} \geq 0$. This is equivalent to $1-u_{1} u_{2} \leq 2\left(1-u_{1}\right)$. Using this inequality in the numerator of (26), we get

$$
2\left(1-u_{1}\right) u_{2}-\left(1-u_{1} u_{2}\right) \phi_{1} B \geq 2\left(1-u_{1}\right)\left(u_{2}-\phi_{1} B\right)>0,
$$

where the second (strict) inequality above follows from our case-(v) assumption that $\phi_{1} B<u_{2}$. This allows us to conclude that $\tilde{h}_{\phi}\left(x^{*}\right)>0$ if $1-2 u_{1}+u_{1} u_{2} \geq 0$.

Now suppose that $1-2 u_{1}+u_{1} u_{2}<0$. Our case-(v) assumption that $B>\phi_{2}$ is equivalent to

$$
\left(1-u_{1}\right) u_{2}>\left(1-u_{2}\right) u_{1} \phi_{1} \phi_{2}
$$

Substituting the definition of $B$ from (25) into the numerator of (26) and using inequality (27), we get

$$
\begin{aligned}
& 2\left(1-u_{1}\right) u_{2}-\left(1-u_{1} u_{2}\right) \phi_{1} B \\
& \quad=\left[2 \sqrt{\left(1-u_{1}\right)\left(1-u_{2}\right) u_{1} u_{2}\left(\phi_{1} \phi_{2}\right)^{-1}}-\left(1-u_{1} u_{2}\right)\right] \phi_{1} B \\
& \quad>-\left(1-2 u_{1}+u_{1} u_{2}\right) \phi_{1} B .
\end{aligned}
$$

The expression in the last line is positive due to our supposition that $1-2 u_{1}+u_{1} u_{2}<0$. This implies that $\tilde{h}_{\phi}\left(x^{*}\right)>0$.

Thus, we get that in case (v), regardless of whether $1-2 u_{1}+u_{1} u_{2} \geq 0$ or $1-2 u_{1}+$ $u_{1} u_{2}<0$, we have $\tilde{h}_{\phi}\left(x^{*}\right)>0$. Then, given that in case $(\mathrm{v})$ function $\tilde{h}_{\phi}(x)$ is convex and $x^{*}$ is its global minimum, we get that $\tilde{h}_{\phi}(x) \geq \tilde{h}_{\phi}\left(x^{*}\right)>0$ for all $x>0$.

Cases (i)-(v) imply that there always exists a $\underline{h}_{\phi}>0$ such that $\tilde{h}_{\phi}(x) \geq \underline{h}_{\phi}$ for all $x>0$. The definitions of $h_{\phi}(x)$ and $\tilde{h}_{\phi}(x)$ then imply that

$$
h_{\phi}(x)=\left(1+\frac{1+u_{2}^{-1} \phi_{1} x}{1+\phi_{1} x} \tilde{h}_{\phi}(x)\right)^{-1} \leq\left(1+\frac{1+u_{2}^{-1} \phi_{1} x}{1+\phi_{1} x} \cdot \underline{h}_{\phi}\right)^{-1} \text {. }
$$

Since $u_{2}<1$, we have

$$
\frac{1+u_{2}^{-1} \phi_{1} x}{1+\phi_{1} x} \cdot \underline{h}_{\phi} \geq \underline{h}_{\phi}
$$

for any $x>0$, and so we get that $h_{\phi}(x) \leq 1-\epsilon_{\phi}$, where $\epsilon_{\phi} \equiv \underline{h}_{\phi} /\left(1+\underline{h}_{\phi}\right)$. Clearly, $0<\epsilon_{\phi}<1$.

Repeating the same proof for $h_{d}(x)$, we can show that there exists $0<\epsilon_{d}<1$ such 
that $h_{d}(x) \leq 1-\epsilon_{d}$. Going back to expressions (24), we get

$$
f_{\phi}(x) \leq\left(1-\epsilon_{d}\right)\left[\phi_{1} \phi_{2}\right]^{-1} f_{d}(x) \text { and } f_{d}(x) \leq\left(1-\epsilon_{\phi}\right)\left[d_{1} d_{2}\right]^{-1} f_{\phi}(x) .
$$

Clearly, if the above inequalities are satisfied for $\epsilon_{d}>0$ and $\epsilon_{\phi}>0$, they are also satisfied for any $\epsilon_{1} \in\left[0, \epsilon_{d}\right]$ and $\epsilon_{2} \in\left[0, \epsilon_{\phi}\right]$ in place of $\epsilon_{d}$ and $\epsilon_{\phi}$, respectively. This completes the proof of Lemma 2.

We now turn to the proof of Proposition 3. We will work with function

$$
V(x) \equiv \ln G-\alpha \ln \left(\frac{\phi_{1}}{\phi_{2}}\right)+(1-\alpha) \ln x+\mu \ln g_{\phi}(x)+\alpha \ln g_{d}(x)
$$

defined in (12) for $x>0$. We have that if $\alpha<1$ then $\lim _{x \rightarrow 0} V(x)=-\infty$ and $\lim _{x \rightarrow \infty} V(x)=$ $\infty$, and if $\alpha>1$ then $\lim _{x \rightarrow 0} V(x)=\infty$ and $\lim _{x \rightarrow \infty} V(x)=-\infty$. Thus, there is at least one positive solution to $V(x)=0$. Our strategy is to derive conditions under which $V(x)$ is monotone, which would imply uniqueness and stability properties.

Differentiating $V(x)$, we get

$$
\frac{d V(x)}{d \ln x}=(1-\alpha)-\mu \cdot\left(1-\phi_{1} \phi_{2}\right) f_{\phi}(x)-\alpha \cdot\left(1-d_{1} d_{2}\right) f_{d}(x),
$$

where

$$
f_{\phi}(x) \equiv \frac{x}{\left(1+\phi_{1} x\right)\left(\phi_{2}+x\right)} \quad \text { and } \quad f_{d}(x) \equiv \frac{x}{\left(1+d_{1} x\right)\left(d_{2}+x\right)} .
$$

Consider $f_{\phi}(x)$. We have

$$
f_{\phi}^{\prime}(x)=\frac{\phi_{1}\left(\left[x_{\phi}^{*}\right]^{2}-x^{2}\right)}{\left(1+\phi_{1} x\right)^{2}\left(\phi_{2}+x\right)^{2}}
$$

where $x_{\phi}^{*} \equiv \sqrt{\phi_{1}^{-1} \phi_{2}}$. We see that $f_{\phi}^{\prime}\left(x_{\phi}^{*}\right)=0$, and $f_{\phi}^{\prime}(x)>0$ for $x<x_{\phi}^{*}$ and $f_{\phi}^{\prime}(x)<0$ for $x>x_{\phi}^{*}$, which means that $x_{\phi}^{*}$ is the global maximum of $f_{\phi}(\cdot)$. Evaluating $f_{\phi}\left(x_{\phi}^{*}\right)$ gives $f_{\phi}\left(x_{\phi}^{*}\right)=\left(1+\sqrt{\phi_{1} \phi_{2}}\right)^{-2}$. Similarly, $f_{d}(x)$ achieves its global maximum at $x_{d}^{*} \equiv \sqrt{d_{1}^{-1} d_{2}}$ with $f_{d}\left(x_{d}^{*}\right)=\left(1+\sqrt{d_{1} d_{2}}\right)^{-2}$. Clearly, $f_{\phi}(x)>0$ and $f_{d}(x)>0$ for all $x>0$, and so we have the following bounds for $f_{\phi}(x)$ and $f_{d}(x)$,

$$
0<f_{\phi}(x) \leq \frac{1}{\left(1+\sqrt{\phi_{1} \phi_{2}}\right)^{2}} \quad \text { and } \quad 0<f_{d}(x) \leq \frac{1}{\left(1+\sqrt{d_{1} d_{2}}\right)^{2}}
$$


Case (i): $\alpha \leq 0$. We have that for any $\alpha$,

$$
\mu=\beta\left(\frac{\alpha}{\varepsilon}+\zeta\right)+(1-\zeta)=\frac{\beta(\psi+\zeta)}{1+\delta}+(1-\zeta)>0 .
$$

If $\alpha \leq 0$ then $\mu \leq \beta \zeta+(1-\zeta) \leq 1$. Using the upper bound (30) for $f_{\phi}(x)$, we get

$$
\left(1-\phi_{1} \phi_{2}\right) f_{\phi}(x) \leq \frac{1-\sqrt{\phi_{1} \phi_{2}}}{1+\sqrt{\phi_{1} \phi_{2}}}<1 .
$$

Thus, $\mu \cdot\left(1-\phi_{1} \phi_{2}\right) f_{\phi}(x)<1$ and so

$$
\frac{d V(x)}{d \ln x}>-\alpha \cdot\left[1+\left(1-d_{1} d_{2}\right) f_{d}(x)\right] .
$$

If $d_{1} d_{2} \leq 1$, then we immediately get that $d V(x) / d \ln x>0$. If $d_{1} d_{2}>1$ then we can use the upper bound (30) for $f_{d}(x)$ to get

$$
\left(d_{1} d_{2}-1\right) f_{d}(x) \leq \frac{\sqrt{d_{1} d_{2}}-1}{\sqrt{d_{1} d_{2}}+1}<1,
$$

which again implies that $d V(x) / d \ln x>0$. Thus, $V(x)$ is a (strictly) increasing function, and so there can be only one solution to $V(x)=0$. Also, the fact that $d V(x) / d \ln x>$ 0 implies that the corresponding equilibrium is globally stable. This proves part (i) of Proposition 3.

Case (ii): $0<\alpha<1$. Suppose that $d_{1} d_{2} \leq 1$. Using the upper bounds (30) on $f_{\phi}(x)$ and $f_{d}(x)$ and the restriction $0<\alpha<1$, we get

$$
\frac{d V(x)}{d \ln x} \geq(1-\alpha)-\mu \cdot \frac{1-\sqrt{\phi_{1} \phi_{2}}}{1+\sqrt{\phi_{1} \phi_{2}}}-\alpha \cdot \frac{1-\sqrt{d_{1} d_{2}}}{1+\sqrt{d_{1} d_{2}}}
$$

From here we see that if

$$
\mu \leq \bar{\mu}_{a} \equiv \frac{1+\sqrt{\phi_{1} \phi_{2}}}{1-\sqrt{\phi_{1} \phi_{2}}} \cdot \frac{1-2 \alpha+\sqrt{d_{1} d_{2}}}{1+\sqrt{d_{1} d_{2}}}
$$

then $V^{\prime}(x)>0$ for all $x>0$, except for, perhaps, one point $x^{*}$ with $V^{\prime}\left(x^{*}\right)=0$. This point $x^{*}$ exists only if $f_{\phi}(x)$ and $f_{d}(x)$ achieve their corresponding global maxima at the same point and, in addition to that, inequality (31) holds with equality. Thus, if inequality (31) holds, then $V(x)$ is increasing in all points except for, maybe, one. This implies that $V(x)$ can intersect the horizontal axis only once and the corresponding equilibrium is generically globally stable. This proves part (ii.a) of Proposition 3. 
Next, suppose that $d_{1} d_{2}>1$. We can use one of the inequalities (23) from Lemma 2, $f_{d}(x) \geq(1-\epsilon)^{-1}\left(\phi_{1} \phi_{2}\right) f_{\phi}(x)$ for any $\epsilon \in[0, \bar{\epsilon}]$, to get

$$
\frac{d V(x)}{d \ln x} \geq(1-\alpha)-\left[\mu \cdot\left(1-\phi_{1} \phi_{2}\right)-(1-\epsilon)^{-1} \alpha \cdot\left(d_{1} d_{2}-1\right) \phi_{1} \phi_{2}\right] f_{\phi}(x),
$$

From here we see that if $\mu \leq \frac{\alpha \cdot\left(d_{1} d_{2}-1\right) \phi_{1} \phi_{2}}{1-\phi_{1} \phi_{2}}$ then $d V(x) / d \ln x \geq 1-\alpha>0$ for all $x$. Thus, in this case $V(x)$ is an increasing function.

Suppose that $\mu>\frac{\alpha \cdot\left(d_{1} d_{2}-1\right) \phi_{1} \phi_{2}}{1-\phi_{1} \phi_{2}}$. Then for all small enough $\epsilon \geq 0$ (including a nonzero measure of $\epsilon \neq 0$ ) we have

$$
\mu-(1-\epsilon)^{-1} \frac{\alpha \cdot\left(d_{1} d_{2}-1\right) \phi_{1} \phi_{2}}{1-\phi_{1} \phi_{2}}>0 .
$$

Then we can apply to (32) the upper bound (30) on $f_{\phi}(x)$ to get that for all small enough $\epsilon \geq 0$

$$
\frac{d V(x)}{d \ln x} \geq(1-\alpha)-\left[\mu-(1-\epsilon)^{-1} \frac{\alpha \cdot\left(d_{1} d_{2}-1\right) \phi_{1} \phi_{2}}{1-\phi_{1} \phi_{2}}\right] \frac{1-\sqrt{\phi_{1} \phi_{2}}}{1+\sqrt{\phi_{1} \phi_{2}}} .
$$

From here we see that if $(1-\epsilon) \mu<\bar{\mu}_{b}$, where

$$
\bar{\mu}_{b} \equiv \alpha \cdot \frac{\left(d_{1} d_{2}-1\right) \phi_{1} \phi_{2}}{1-\phi_{1} \phi_{2}}+(1-\alpha) \cdot \frac{1+\sqrt{\phi_{1} \phi_{2}}}{1-\sqrt{\phi_{1} \phi_{2}}}
$$

then $d V(x) / d \ln x>0$. Since $\mu>0$ for any $\alpha$, if $\mu \leq \bar{\mu}_{b}$ then $(1-\epsilon) \mu<\bar{\mu}_{2}$ for all $\epsilon \in(0,1)$. Observe that $\bar{\mu}_{b}>\frac{\alpha \cdot\left(d_{1} d_{2}-1\right) \phi_{1} \phi_{2}}{1-\phi_{1} \phi_{2}}$. Therefore, we get that if $d_{1} d_{2}>1$ and $\mu \leq \bar{\mu}_{b}$ then $d V(x) / d \ln x>0$ for all $x>0$, and so there can be only one positive solution to $V(x)=0$. Also, the corresponding equilibrium is globally stable. This proves part (ii.b) of Proposition 3.

Case (iii): $\alpha>1$. The proof for this case is very similar to the proof for case (ii).

Since we have $\mu>0$ and $\alpha>1$, expression (28) for $d V(x) / d \ln x$ immediately implies that if $d_{1} d_{2} \leq 1$ then $d V(x) / d \ln x<0$ for all $x$. So, in this case $V(x)$ is a decreasing function and there can be only one positive solution to $V(x)=0$. The corresponding equilibrium is unstable. This proves part (iii.a) of Proposition 3.

Suppose now that $d_{1} d_{2}>1$. We can use one of the inequalities (23) from Lemma 2, $f_{\phi}(x) \geq(1-\epsilon)^{-1}\left(d_{1} d_{2}\right) f_{d}(x)$ for any $\epsilon \in[0, \bar{\epsilon}]$, to get

$$
\frac{d V(x)}{d \ln x} \leq(1-\alpha)-\left[(1-\epsilon)^{-1} \mu \cdot\left(1-\phi_{1} \phi_{2}\right) d_{1} d_{2}-\alpha \cdot\left(d_{1} d_{2}-1\right)\right] f_{d}(x)
$$

From here we see that if $\mu \geq \frac{\alpha \cdot\left(d_{1} d_{2}-1\right)}{\left(1-\phi_{1} \phi_{2}\right) d_{1} d_{2}}$ then $d V(x) / d \ln x \leq 1-\alpha<0$ for all $x$. Thus, 
in this case $V(x)$ is a decreasing function.

Suppose that $\mu<\frac{\alpha \cdot\left(d_{1} d_{2}-1\right)}{\left(1-\phi_{1} \phi_{2}\right) d_{1} d_{2}}$. Then for all small enough $\epsilon \geq 0$ (including a nonzero measure of $\epsilon \neq 0$ ) we have

$$
(1-\epsilon)^{-1} \mu-\frac{\alpha \cdot\left(d_{1} d_{2}-1\right)}{\left(1-\phi_{1} \phi_{2}\right) d_{1} d_{2}}<0
$$

Then we can apply to (33) the upper bound (30) on $f_{d}(x)$ to get that for all small enough $\epsilon \geq 0$

$$
\frac{d V(x)}{d \ln x} \leq(1-\alpha)-\left[(1-\epsilon)^{-1} \mu \cdot \frac{\left(1-\phi_{1} \phi_{2}\right) d_{1} d_{2}}{d_{1} d_{2}-1}-\alpha\right] \frac{\sqrt{d_{1} d_{2}}-1}{\sqrt{d_{1} d_{2}}+1} .
$$

From here we see that if $(1-\epsilon)^{-1} \mu>\overline{\bar{\mu}}$, where

$$
\overline{\bar{\mu}} \equiv \frac{d_{1} d_{2}-1}{\left(1-\phi_{1} \phi_{2}\right) d_{1} d_{2}} \cdot \frac{1-2 \alpha+\sqrt{d_{1} d_{2}}}{\sqrt{d_{1} d_{2}}-1}
$$

then $d V(x) / d \ln x<0$. Since $\mu>0$ for any $\alpha$, if $\mu \geq \overline{\bar{\mu}}$ then $(1-\epsilon)^{-1} \mu>\overline{\bar{\mu}}$ for all $\epsilon \in(0,1)$. Observe that $\overline{\bar{\mu}}<\frac{\alpha \cdot\left(d_{1} d_{2}-1\right)}{\left(1-\phi_{1} \phi_{2}\right) d_{1} d_{2}}$. Therefore, we get that if $d_{1} d_{2}>1$ and $\mu \geq \overline{\bar{\mu}}$ then $V(x)$ is a decreasing function, and so there can be only one positive solution to $V(x)=0$. The corresponding equilibrium is unstable. This proves part (iii.b) of Proposition 3.

\section{B.5.1 Corollary: Uniqueness under Small Trade Costs or Small $\alpha \in(0,1)$}

Corollary 1. Assume that $0<\alpha<1$ and $\phi_{1} \phi_{2}<1$. The economy has a unique regular (generically globally stable) equilibrium if $\phi_{1} \phi_{2}$ is close enough to 1 or $\alpha$ is low enough.

Proof. Suppose that $\phi_{1} \phi_{2} \lesssim 1$, where "ミ" means approximately equal but less than. Since $d_{1} d_{2} \leq 1$ is equivalent to

$$
\frac{\gamma \bar{\gamma} \zeta^{2} \bar{\beta}^{2}}{(1-\zeta \bar{\gamma} \bar{\beta})(1-\zeta \gamma \bar{\beta})} \leq \phi_{1} \phi_{2} \leq 1,
$$

having $\phi_{1} \phi_{2} \lesssim 1$ implies that $d_{1} d_{2}<1$. Also, we have $d_{1} d_{2} \rightarrow 1$ as $\phi_{1} \rightarrow 1$ and $\phi_{2} \rightarrow 1$. Thus, we have that $\phi_{1} \phi_{2} \lesssim 1$ implies $d_{1} d_{2} \lesssim 1$. Then, to establish uniqueness under $0<\alpha<1$ we need to show that the condition $\mu \leq \frac{1+\sqrt{\phi_{1} \phi_{2}}}{1-\sqrt{\phi_{1} \phi_{2}}} \cdot \frac{1-2 \alpha+\sqrt{d_{1} d_{2}}}{1+\sqrt{d_{1} d_{2}}}$ in part (ii.a) of Proposition 3 holds. With $\phi_{1} \phi_{2} \lesssim 1$ we have that $\frac{1+\sqrt{\phi_{1} \phi_{2}}}{1-\sqrt{\phi_{1} \phi_{2}}}$ is positive and large, and so it is enough to show that $1-2 \alpha+\sqrt{d_{1} d_{2}}$ is positive. This is equivalent to $\frac{1+\sqrt{d_{1} d_{2}}}{2}>\alpha$. For any $\alpha$ there are always trade costs that are low enough so that $d_{1} d_{2}$ is close to 1 and, consequently, $\frac{1+\sqrt{d_{1} d_{2}}}{2}$ is close enough to 1 . Since $\alpha<1$, then the inequality follows. 
Consider now $\alpha \gtrsim 0$. Suppose first that $d_{1} d_{2} \leq 1$. We have that $\mu \approx \beta \zeta+(1-\zeta) \leq 1$ and $\bar{\mu}_{a} \approx \frac{1+\sqrt{\phi_{1} \phi_{2}}}{1-\sqrt{\phi_{1} \phi_{2}}}>1$, and thus $\mu<\bar{\mu}_{a}$ for low enough $\alpha>0$. Part (ii.a) of Proposition 3 then implies uniqueness for $d_{1} d_{2} \leq 1$ and $\alpha \gtrsim 0$. Now suppose that $d_{1} d_{2}>1$. Then, $\bar{\mu}_{b} \approx \frac{1+\sqrt{\phi_{1} \phi_{2}}}{1-\sqrt{\phi_{1} \phi_{2}}}>1$ for $\alpha \gtrsim 0$, while we still have $\mu \approx \beta \zeta+(1-\zeta) \leq 1$. Thus $\mu<\bar{\mu}_{b}$ and part (ii.b) of Proposition 3 then implies uniqueness for $d_{1} d_{2}>1$ and $\alpha \gtrsim 0$.

\section{B.5.2 Corollary: Uniqueness for $\alpha \in(0,1)$ and Small $\beta$}

Corollary 2. Consider the case without an input-output loop. If $0<\alpha<1$ then there exists a small enough $\beta>0$ such that the equilibrium is unique for all $\phi_{1} \leq 1$ and $\phi_{2} \leq 1$.

Proof. Assume that $\zeta=1$. Denote $\phi=\phi_{1} \phi_{2}$ and let

$$
f_{d}(\phi, \beta) \equiv d_{1} d_{2}=1+\frac{\left(\phi-f_{\phi}(\beta)\right)(\phi-1)}{\phi},
$$

with

$$
f_{\phi}(\beta) \equiv \frac{\gamma \bar{\gamma} \bar{\beta}^{2}}{(1-\gamma \bar{\beta})(1-\bar{\gamma} \bar{\beta})} .
$$

It is straightforward to check that $f_{\phi}(\beta) \in[0,1]$ for $\beta \in[0,1]$. Thus, we have that $f_{d}(\phi, \beta) \leq 1$ for $\phi \in\left[f_{\phi}(\beta), 1\right]$ and $f_{d}(\phi, \beta)>1$ for $\phi<f_{\phi}(\beta)$. Observe that $f_{\phi}(\beta)$ is a decreasing function of $\beta$ and $f_{\phi}(\beta)=1$ for $\beta=0$. Thus, we can make $f_{\phi}(\beta)$ arbitrary close 1 (from below) by choosing $\beta$ close enough to 0 .

Next, given that

$$
\frac{\partial f_{d}(\phi, \beta)}{\partial \phi}=\phi^{-2}\left(\phi^{2}-f_{\phi}(\beta)\right)
$$

we have that $f_{d}(\phi, \beta)$ is a convex function of $\phi$ that achieves its minimum at $\phi=\sqrt{f_{\phi}(\beta)}>$ $f_{\phi}(\beta)$. Simple algebra reveals that

$$
f_{d}\left(\sqrt{f_{\phi}(\beta)}, \beta\right)=\left(2-\sqrt{f_{\phi}(\beta)}\right) \sqrt{f_{\phi}(\beta)} \geq \sqrt{f_{\phi}(\beta)} .
$$

Thus, we have that $f_{d}(\phi, \beta) \geq \sqrt{f_{\phi}(\beta)}$ for all $\phi \in(0,1]$ and $\beta \in[0,1]$.

Let us now show that we can choose a small enough $\beta>0$ such that equilibrium is unique for all $\phi_{1}, \phi_{2} \in(0,1]$. If $\phi_{1}=\phi_{2}=1$ then equilibrium is unique for any $\beta \in(0,1]$ thanks to Proposition 2. Suppose that either $\phi_{1}<1$ or $\phi_{2}<1$ or both, which implies that $\phi \in(0,1)$. Let $\beta_{1}>0$ be such that $\left[f_{\phi}\left(\beta_{1}\right)\right]^{\frac{1}{4}}=\alpha$. Then $\left[f_{d}(\phi, \beta)\right]^{\frac{1}{2}} \geq \alpha$ for all $\beta \in\left(0, \beta_{1}\right]$ and $\phi \in(0,1)$, which, combined with the fact that $f_{d}(\phi, \beta) \leq 1$ for all $\beta \in(0,1)$ and 
$\phi \in\left[f_{\phi}(\beta), 1\right]$, implies that for all $\beta \in\left(0, \beta_{1}\right]$ and $\phi \in\left[f_{\phi}(\beta), 1\right)$ and we have

$$
\bar{\mu}_{a}=\frac{1+\sqrt{\phi}}{1-\sqrt{\phi}} \cdot \frac{1-2 \alpha+\left[f_{d}(\phi, \beta)\right]^{\frac{1}{2}}}{1+\left[f_{d}(\phi, \beta)\right]^{\frac{1}{2}}} \geq \hat{\bar{\mu}}_{a} \equiv \frac{1+\alpha^{2}}{1-\alpha^{2}} \cdot \frac{1-\alpha}{2}>0
$$

where $\bar{\mu}_{a}$ is the constant used in part (ii.a) of Proposition 3. Here we also used the fact that $f_{\phi}(\beta) \geq f_{\phi}\left(\beta_{1}\right)=\alpha^{4}$ for all $\beta \in\left(0, \beta_{1}\right]$.

Next, for any $\beta \in(0,1)$ and $\phi \in\left(0, f_{\phi}(\beta)\right)$ we have $f_{d}(\phi, \beta)>1$ and

$$
\bar{\mu}_{b}=\alpha \cdot \frac{\left(d_{1} d_{2}-1\right) \phi_{1} \phi_{2}}{1-\phi_{1} \phi_{2}}+(1-\alpha) \cdot \frac{1+\sqrt{\phi_{1} \phi_{2}}}{1-\sqrt{\phi_{1} \phi_{2}}} \geq \hat{\bar{\mu}}_{b} \equiv 1-\alpha>0
$$

where $\bar{\mu}_{b}$ is the constant used in part (ii.b) of Proposition 3.

Finally, $\mu=\beta(\chi+1)$ is an increasing function of $\beta$ and thus we can choose $\beta_{2} \in\left(0, \beta_{1}\right]$ such that $\beta_{2}(\chi+1) \leq \max \left\{\hat{\bar{\mu}}_{a}, \hat{\bar{\mu}}_{b}\right\}$. This value of $\beta_{2}>0$ is such that the equilibrium is unique for all $\phi_{1} \phi_{2} \in(0,1)$. Indeed, if $\phi_{1} \phi_{2} \in\left[f_{\phi}\left(\beta_{2}\right), 1\right)$ then $d_{1} d_{2} \leq 1$ and $\mu \leq \hat{\mu}_{a} \leq \bar{\mu}_{a^{\prime}}$ and so equilibrium is unique by virtue of part (ii.a) of Proposition 3. If, on the other hand, $\phi_{1} \phi_{2} \in\left(0, f_{\phi}\left(\beta_{2}\right)\right)$ then $d_{1} d_{2}>1$ and $\mu \leq \hat{\bar{\mu}}_{b} \leq \bar{\mu}_{b}$, and so equilibrium is unique by virtue of part (ii.b) of Proposition 3.

\section{B.6 Proof of Proposition 4}

We break the proof of Proposition 4 into three Lemmas. Lemma 3 establishes sufficiency of conditions (a)-(c) of Proposition 4 for uniqueness of a regular equilibrium, Lemma 4 deals with existence, and Lemma 5 deals with stability.

Lemma 3. Assume that $\alpha=1, \phi_{1} \phi_{2}<1$, and any of the conditions (a)-(c) of Proposition 4 holds. Then the economy has at most one regular equilibrium.

Proof. For $\alpha=1$ function $V(x)$ defined in (12) becomes

$$
V(x)=\ln G-\ln \left(\frac{\phi_{1}}{\phi_{2}}\right)+\mu \ln g_{\phi}(x)+\ln g_{d}(x) .
$$

Let us introduce the change of variables

$$
z=\left(\frac{1+\phi_{1} x}{\phi_{2}+x}\right)^{1+\mu}
$$


which implies that

$$
x=\frac{1-\phi_{2} z^{\frac{1}{1+\mu}}}{z^{\frac{1}{1+\mu}}-\phi_{1}} .
$$

Observe that $z$ ranges from $\phi_{2}^{-(1+\mu)}$ to $\phi_{1}^{1+\mu}$ as $x$ ranges from 0 to $\infty$.

Given the definitions of $z, d_{1}$, and $d_{2}$, and using the assumption $\phi_{1} \phi_{2}<1$, we can write

$$
\frac{1+d_{1} x}{d_{2}+x}=\frac{\phi_{1}}{\phi_{2}} \cdot \frac{\zeta \gamma \bar{\beta}+(1-\zeta \gamma \bar{\beta}) \phi_{2} z^{\frac{1}{1+\mu}}}{(1-\zeta \bar{\gamma} \bar{\beta}) \phi_{1}+\zeta \bar{\gamma} \bar{\beta} z^{\frac{1}{1+\mu}}} .
$$

Substituting this and the definition of $z$ into $V(x)$, and after doing some algebra, we can write equation $V(x)=0$ as $\tilde{V}(z)=0$, where

$$
\begin{aligned}
\tilde{V}(z) & \equiv\left((1-\zeta \bar{\gamma} \bar{\beta}) \phi_{1}+\zeta \bar{\gamma} \bar{\beta} z^{\frac{1}{1+\mu}}\right) \cdot\left\{1-\exp V\left(\frac{1-\phi_{2} z^{\frac{1}{1+\mu}}}{z^{\frac{1}{1+\mu}}-\phi_{1}}\right)\right\} \\
& =\zeta \bar{\gamma} \bar{\beta} z^{\frac{1}{1+\mu}}-\zeta \gamma \bar{\beta} G z^{\frac{\mu}{1+\mu}}-(1-\zeta \gamma \bar{\beta}) \phi_{2} G z+(1-\zeta \bar{\gamma} \bar{\beta}) \phi_{1} .
\end{aligned}
$$

Formally, equation $\tilde{V}(z)=0$ is equivalent to equation $V(x)=0$ only for $z \in\left(\phi_{1}^{1+\mu}, \phi_{2}^{-(1+\mu)}\right)$. At the same time, function $\tilde{V}(z)$ is defined for any $z \geq 0$. Moreover, $\tilde{V}(z)=0$ has at least one solution for some $z>0$, because $\tilde{V}(0)>0$ and $\lim _{z \rightarrow \infty} \tilde{V}(z)=-\infty$, while equation $V(x)=0$ might have no positive solutions. In the proof of the current lemma, we analyze multiplicity of solutions of equation $\tilde{V}(z)=0$ for all $z>0$.

If $\bar{\beta}=0$ then we can explicitly find that the solution of equation $\tilde{V}(z)=0$ is $\tilde{z}=$ $\phi_{1} \phi_{2}^{-1} G^{-1}$. If $\gamma=0$ and $\bar{\beta}>0$ then $\tilde{V}(z)$ is a concave function achieving its maximum at a positive $z^{*}$. Then, given that $\tilde{V}(0)>0$ and $\lim _{z \rightarrow \infty} \tilde{V}(z)=-\infty$, we conclude that $\tilde{V}(z)$ intersects the horizontal axis only once. If $\gamma=1$ and $\bar{\beta}>0$ then $\tilde{V}(z)$ is a strictly decreasing function with $\tilde{V}(0)>0$ and $\lim _{z \rightarrow \infty} \tilde{V}(z)=-\infty$. Hence, again, $\tilde{V}(z)$ intersects the horizontal axis only once. This proves the current lemma for the case when any of the conditions in part (a) of Proposition 4 holds.

If $\mu=1$ then equation $\tilde{V}(z)=0$ turns into the quadratic equation,

$$
(1-\zeta \gamma \bar{\beta}) \phi_{2} G z-(\bar{\gamma}-\gamma G) \zeta \bar{\beta} z^{\frac{1}{2}}-(1-\zeta \bar{\gamma} \bar{\beta}) \phi_{1}=0,
$$

which has a unique positive solution. This proves the case with $\mu=1$.

In what follows, we assume that $\bar{\beta} \neq 0$ and $0<\gamma<1$ and consider the cases $\mu<1$ 
and $\mu>1$. We have

$$
\begin{aligned}
\tilde{V}^{\prime}(z) & =\frac{1}{1+\mu} \zeta \bar{\gamma} \bar{\beta} z^{-\frac{\mu}{1+\mu}}-\frac{\mu}{1+\mu} \zeta \gamma \bar{\beta} G z^{-\frac{1}{1+\mu}}-(1-\zeta \gamma \bar{\beta}) \phi_{2} G \\
\tilde{V}^{\prime \prime}(z) & =\frac{\mu \zeta \gamma \bar{\beta} G}{(1+\mu)^{2}} z^{-\frac{\mu}{1+\mu}-1}\left(z^{-\frac{1-\mu}{1+\mu}}-\bar{z}_{0}^{-\frac{1-\mu}{1+\mu}}\right)
\end{aligned}
$$

where

$$
\bar{z}_{0} \equiv\left(\frac{\gamma}{\bar{\gamma}} G\right)^{\frac{1+\mu}{1-\mu}}
$$

Consider the case with $\mu>1$. In this case, $\tilde{V}^{\prime \prime}(z)<0$ for $z<\bar{z}_{0}$, and $\tilde{V}^{\prime \prime}(z)>0$ for $z>\bar{z}_{0}$. Thus, $\tilde{V}^{\prime}(z)$ is a convex function with $\bar{z}_{0}$ being its minimum. We have

$$
\tilde{V}^{\prime}\left(\bar{z}_{0}\right)=-\frac{\mu-1}{\mu+1} \zeta \bar{\beta} \gamma^{\frac{\mu}{\mu-1}} \bar{\gamma}^{-\frac{1}{\mu-1}} G^{\frac{\mu}{\mu-1}}-(1-\zeta \gamma \bar{\beta}) \phi_{2} G .
$$

Clearly, $\tilde{V}^{\prime}\left(\bar{z}_{0}\right)<0$, which, together with $\lim _{z \rightarrow 0} \tilde{V}^{\prime}(z)=\infty$ and $\lim _{z \rightarrow \infty} \tilde{V}^{\prime}(z)<0$, implies that $\tilde{V}^{\prime}(z)$ intersects the horizontal axis only once for some $z_{1}^{*}<\bar{z}_{0}$. Therefore, $\tilde{V}(z)$ is increasing for $z<z_{1}^{*}$ and decreasing for $z>z_{1}^{*}$. Then, given that $\tilde{V}(0)>0$ and $\lim _{z \rightarrow \infty} \tilde{V}(z)=-\infty$, we have that $\tilde{V}(z)$ intersect the horizontal axis only once for some $\tilde{z}>z_{1}^{*}$. This proves the current lemma for the case when condition in part (b) of Proposition 4 holds.

Now consider the case with $\mu<1$. In this case, $\tilde{V}^{\prime \prime}(z)>0$ for $z<\bar{z}_{0}$ and $\tilde{V}^{\prime \prime}(z)<0$ for $z>\bar{z}_{0}$. Thus, $\tilde{V}^{\prime}(z)$ is a concave function with $\bar{z}_{0}$ being its maximum. Evaluating $\tilde{V}^{\prime}\left(\bar{z}_{0}\right)$ we get the same expression (38) as before. It then is straightforward to check that if

$$
\phi_{2}>\tilde{\phi}_{2} \equiv \frac{1-\mu}{1+\mu} \cdot \frac{\zeta \gamma \bar{\beta}}{1-\zeta \gamma \bar{\beta}}\left(\frac{\gamma}{\bar{\gamma}}\right)^{-\frac{1}{1-\mu}} G^{-\frac{1}{1-\mu}},
$$

then $\tilde{V}^{\prime}\left(\bar{z}_{0}\right)<0$, which implies that $\tilde{V}^{\prime}(z)<0$ for all $z$. This means that in this case $\tilde{V}(z)$ is a decreasing function that intersects the horizontal axis only once.

The case with $\phi_{2}=\tilde{\phi}_{2}$ (and $\mu<1$ ) is similar to the case with $\phi_{2}>\tilde{\phi}_{2}$ with the only difference that if $\phi_{2}=\tilde{\phi}_{2}$ then $\tilde{V}^{\prime}\left(\bar{z}_{0}\right)=0$ and $\tilde{V}(z)$ is decreasing for all $z \neq \bar{z}_{0}$. Clearly, equation $\tilde{V}(z)=0$ has a unique solution in this case as well. This proves the current lemma for the case when the condition with $\phi_{2} \geq \tilde{\phi}_{2}$ in part (c) of Proposition 4 holds.

Finally, consider

$$
\tilde{V}(z) \equiv-G^{-1} z \tilde{V}\left(z^{-1}\right)=\zeta \gamma \bar{\beta} z^{\frac{1}{1+\mu}}-\zeta \bar{\gamma} \bar{\beta} G^{-1} z^{\frac{\mu}{1+\mu}}-(1-\zeta \bar{\gamma} \bar{\beta}) \phi_{1} G^{-1} z+(1-\zeta \gamma \bar{\beta}) \phi_{2} .
$$


Clearly, $\tilde{V}(z)$ and $\tilde{V}(z)$ have the same number of positive solutions. Repeating the above analysis for $\tilde{V}(z)$ instead of $\tilde{V}(z)$, we get that $\tilde{V}(z)$ intersects the horizontal axis once and only once if

$$
\phi_{1} \geq \tilde{\phi}_{1} \equiv \frac{1-\mu}{1+\mu} \cdot \frac{\zeta \bar{\gamma} \bar{\beta}}{1-\zeta \bar{\gamma} \bar{\beta}}\left(\frac{\gamma}{\bar{\gamma}}\right)^{\frac{1}{1-\mu}} G^{\frac{1}{1-\mu}} .
$$

This completes the proof the current lemma for the case when part (c) of Proposition 4 holds.

Lemma 4. Assume that $\alpha=1, \phi_{1} \phi_{2}<1$, and any of the conditions (a)-(c) of Proposition 4 holds. Then the economy has a regular equilibrium only if condition (14) holds.

Proof. As argued in Lemma 3, any solution $\tilde{z}$ to equation $\tilde{V}(z)=0$ will be a solution to the original equation $V(x)=0$ only if $\tilde{z} \in\left(\phi_{1}^{1+\mu}, \phi_{2}^{-(1+\mu)}\right)$. In Lemma 3 we have established that if any of the conditions (a)-(c) of Proposition 4 holds, then there is a unique positive solution $\tilde{z}$ to equation $\tilde{V}(z)=0$. At the same time, regardless of which condition (a)-(c) of Proposition 4 holds, we have $\lim _{z \rightarrow 0} \tilde{V}(z)>0$ and $\lim _{z \rightarrow \infty} \tilde{V}(z)=-\infty$. Thus, we necessarily have that $\tilde{V}(z)>0$ for all $z<\tilde{z}$ and $\tilde{V}(z)<0$ for all $z>\tilde{z}$. Therefore, in all cases with the unique solution $\tilde{z}$ to $\tilde{V}(z)=0$, we have $\tilde{z} \in\left(\phi_{1}^{1+\mu}, \phi_{2}^{-(1+\mu)}\right)$ if and only if $\tilde{V}\left(\phi_{1}^{1+\mu}\right)>0>\tilde{V}\left(\phi_{2}^{-(1+\mu)}\right)$, which, after some algebra, gives

$$
\zeta \bar{\gamma} \bar{\beta} \phi_{2}^{\mu-1}+(1-\zeta \bar{\gamma} \bar{\beta}) \phi_{1} \phi_{2}^{\mu}<G<\left[\zeta \gamma \bar{\beta} \phi_{1}^{\mu-1}+(1-\zeta \gamma \bar{\beta}) \phi_{1}^{\mu} \phi_{2}\right]^{-1} .
$$

This is condition (14) in the main text.

Lemma 5. Assume that $\alpha=1$ and $\phi_{1} \phi_{2}<1$. Also assume that condition (14) as well as any of the conditions (a)-(c) of Proposition 4 hold. Then the unique regular equilibrium is generically unstable.

Proof. In this lemma we used the notation introduced in Lemma 3.

The proof in Lemma 3 implies that for the unique solution $\tilde{z}$ to $\tilde{V}(z)=0$ we have $\tilde{V}^{\prime}(\tilde{z})<0$ if $\bar{\beta}=0, \gamma=0, \gamma=1$, or $\mu \geq 1$. In the case with $\mu<1$, the proof of Lemma 3 implies that $\tilde{V}^{\prime}(\tilde{z})<0$ if $\phi_{2}>\tilde{\phi}_{2}$, or if $\phi_{2}=\tilde{\phi}_{2}$ and $\tilde{z} \neq \bar{z}_{0}$. If $\mu<1, \phi_{2}=\tilde{\phi}_{2}$, and $\tilde{z}=\bar{z}_{0}$ then $V^{\prime}(\tilde{z})=0$. Consider now the case with $\mu<1$ and $\phi_{1} \geq \tilde{\phi}_{1}$. We have $\tilde{V}(z)=-G z \tilde{V}\left(z^{-1}\right)$ and so $\tilde{V}^{\prime}(\tilde{z})=G \tilde{z}^{-1} \tilde{V}^{\prime}\left(\tilde{z}^{-1}\right) \leq 0$, similarly to the case with $\mu<1$ and $\phi_{2} \geq \tilde{\phi}_{2}$. Thus, under any of the conditions (a)-(c) of Proposition 4 , we have that generically $V^{\prime}(\tilde{z})<0$.

Next, observe that

$$
\frac{d \ln z}{d \ln x}=-\frac{(1+\mu)\left(1-\phi_{1} \phi_{2}\right) x}{\left(1+\phi_{1} x\right)\left(\phi_{2}+x\right)}<0,
$$


and so $z$ is a decreasing function of $x$ under our assumption that $\phi_{1} \phi_{2}<1$.

Finally, suppose that condition (14) holds, so that $z \in\left(\phi_{1}^{1+\mu}, \phi_{2}^{-(1+\mu)}\right)$ and there is a corresponding solution $\tilde{x}>0$ to $V(x)=0$. We have

$$
\frac{d \tilde{V}(\tilde{z})}{d \ln z}=-\left((1-\zeta \bar{\gamma} \bar{\beta}) \phi_{1}+\zeta \bar{\gamma} \bar{\beta} \tilde{z}^{\frac{1}{1+\mu}}\right) \cdot \frac{d V(\tilde{x})}{d \ln x} \cdot \frac{d \ln x(\tilde{z})}{d \ln z},
$$

where $x(z)=\left(1-\phi_{2} z^{\frac{1}{1+\mu}}\right) /\left(z^{\frac{1}{1+\mu}}-\phi_{1}\right)$. Given that $d \ln z(\tilde{x}) / d \ln x<0$ is equivalent to $d \ln x(\tilde{z}) / d \ln z<0$, we get

$$
\frac{d V(\tilde{x})}{d \ln x}=-\frac{1}{(1-\zeta \bar{\gamma} \bar{\beta}) \phi_{1}+\zeta \bar{\gamma} \bar{\beta} \tilde{z}^{\frac{1}{1+\mu}}} \cdot \frac{d \tilde{V}(\tilde{z})}{d \ln z} \cdot \frac{d \ln z(\tilde{x})}{d \ln x} .
$$

This implies that $d V(\tilde{x}) / d \ln x<0$ if and only if $d V(\tilde{z}) / d \ln z<0$. Thus, if condition (14) as well as any of the conditions (a)-(c) of Proposition 4 hold, then the unique regular equilibrium is generically unstable.

\section{Proof of Proposition 5}

In the case with $\beta=1$, expressions for $d_{1}$ and $d_{2}$ collapse to $d_{1}=\phi_{1}$ and $d_{2}=\phi_{2}$, and function $V(x)$ becomes

$$
V(x)=\ln G-\alpha \ln \frac{\phi_{1}}{\phi_{2}}+(1-\alpha) \ln x+(\alpha+\mu) \ln \frac{1+\phi_{1} x}{\phi_{2}+x} .
$$

We have

$$
V^{\prime}(x)=\frac{(1-\alpha)\left(1+\phi_{1} x\right)\left(\phi_{2}+x\right)-(\alpha+\mu)\left(1-\phi_{1} \phi_{2}\right) x}{\left(1+\phi_{1} x\right)\left(\phi_{2}+x\right) x} .
$$

Thus, $V^{\prime}(x) \geq 0$ if and only if $W(x) \geq 0$, where

$$
W(x) \equiv(1-\alpha) \phi_{1} x^{2}+\left[(\mu+1) \phi_{1} \phi_{2}-(\mu+2 \alpha-1)\right] x+(1-\alpha) \phi_{2} .
$$

Observe that $W(x)$ is a quadratic polynomial in $x$. Denoting by $\widetilde{D}$ the discriminant of equation $W(x)=0$, we can write

$$
\widetilde{D} \equiv(\mu+1)^{2}\left(1-\phi_{1} \phi_{2}\right)\left(\eta^{2}-\phi_{1} \phi_{2}\right),
$$


where $\eta \equiv \frac{\mu+2 \alpha-1}{\mu+1}$. Observe that $0<\eta<1$ for $0<\alpha<1$. If $\eta<\sqrt{\phi_{1} \phi_{2}} \leq 1$, then $W(x)>0$ for all $x$ and so $V(x)$ is an increasing function of $x$ for all $x>0$. Then, given that $\lim _{x \rightarrow 0} V(x)=-\infty$ and $\lim _{x \rightarrow \infty} V(x)=\infty$, we get that for $\eta<\phi_{1} \phi_{2}<1$ function $V(x)$ intersects the horizontal axis $x=0$ once and only once. The corresponding equilibrium is globally stable.

The case with $\eta=\sqrt{\phi_{1} \phi_{2}}$ is similar to the case with $\eta<\sqrt{\phi_{1} \phi_{2}}<1$ with the only difference that $V(x)$ is an increasing function for all $x>0$ except for one $x=x^{*}$ such that $V^{\prime}\left(x^{*}\right)=0$. In this case $V(x)$ also intersects the horizontal axis once and only once. The corresponding equilibrium is generically globally stable.

Consider the case with $\eta>\sqrt{\phi_{1} \phi_{2}}$. In this case equation $W(x)=0$ has two distinct positive solutions

$$
x_{1}^{*}=\frac{(\mu+1)\left(\eta-\phi_{1} \phi_{2}\right)-\sqrt{\widetilde{D}}}{2(1-\alpha) \phi_{1}} \quad \text { and } \quad x_{2}^{*}=\frac{(\mu+1)\left(\eta-\phi_{1} \phi_{2}\right)+\sqrt{\widetilde{D}}}{2(1-\alpha) \phi_{1}} .
$$

We have that $W(x)>0$ for $x<x_{1}^{*}$ and $x>x_{2}^{*}$, and $W(x)<0$ for $x_{1}^{*}<x<x_{2}^{*}$. Thus, function $V(x)$ is increasing for $x<x_{1}^{*}$ and $x>x_{2}^{*}$, and $V(x)$ is decreasing for $x_{1}^{*}<x<x_{2}^{*}$. Given that $\lim _{x \rightarrow 0} V(x)=-\infty$ and $\lim _{x \rightarrow \infty} V(x)=\infty$, we have that if $V\left(x_{1}^{*}\right)<0$ or $V\left(x_{2}^{*}\right)>0$ then function $V(x)$ intersects the horizontal axis only once, and the corresponding equilibrium is locally stable.

Denote

$$
\begin{aligned}
\Gamma_{1} & \equiv \frac{G(\mu+1)^{\mu+1}\left[\phi_{1} \phi_{2}^{\mu}\right]^{-1}}{2^{\mu+1}(1-\alpha)^{1-\alpha}(\mu+\alpha)^{\mu+\alpha}}\left(\eta-\phi_{1} \phi_{2}-D\right)^{1-\alpha}\left(\eta+\phi_{1} \phi_{2}+D\right)^{\mu+\alpha}, \\
\Gamma_{2} & \equiv \frac{G(\mu+1)^{\mu+1}\left[\phi_{1} \phi_{2}^{\mu}\right]^{-1}}{2^{\mu+1}(1-\alpha)^{1-\alpha}(\mu+\alpha)^{\mu+\alpha}}\left(\eta-\phi_{1} \phi_{2}+D\right)^{1-\alpha}\left(\eta+\phi_{1} \phi_{2}-D\right)^{\mu+\alpha},
\end{aligned}
$$

where

$$
D \equiv \sqrt{\left(1-\phi_{1} \phi_{2}\right)\left(\eta^{2}-\phi_{1} \phi_{2}\right)}
$$

Then condition $V\left(x_{1}^{*}\right)<0$ can be written as $\Gamma_{1}<1$, while condition $V\left(x_{2}^{*}\right)>0$ can be written as $\Gamma_{2}>1$.

If $V\left(x_{1}^{*}\right)=0$ then the horizontal axis is tangent to function $V(x)$ at $x_{1}^{*}$ and also $V(x)$ intersects the horizontal axis at some point $\tilde{x}>x_{1}^{*}$ such that $V^{\prime}(\tilde{x})>0$. Thus, in this case equation $V(x)=0$ has two solutions. The equilibrium corresponding to $x_{1}^{*}$ is neither stable nor unstable, while the equilibrium corresponding to $\tilde{x}$ is locally stable. Similarly, if $V\left(x_{2}^{*}\right)=0$ then the horizontal axis is tangent to function $V(x)$ at $x_{2}^{*}$ and also $V(x)$ 
intersects the horizontal axis at some point $\tilde{x}<x_{1}^{*}$ such that $V^{\prime}(\tilde{x})>0$. In this case equation $V(x)=0$ also has two solutions. The equilibrium corresponding to $x_{2}^{*}$ is neither stable nor unstable, while the equilibrium corresponding to $\tilde{x}$ is locally stable. Observe that condition $V\left(x_{1}^{*}\right)=0$ can be written as $\Gamma_{1}=1$, while condition $V\left(x_{2}^{*}\right)=0$ can be written as $\Gamma_{2}=1$.

Finally, if $V\left(x_{1}^{*}\right)>0$ and $V\left(x_{2}^{*}\right)<0$, which can be written as $\Gamma_{1}>1$ and $\Gamma_{2}<1$, then function $V(x)$ intersects the horizontal axis three times at some points $\tilde{x}_{1}, \tilde{x}_{2}$, and $\tilde{x}_{3}$ such that $\tilde{x}_{1}<x_{1}^{*}<\tilde{x}_{2}<x_{2}^{*}<\tilde{x}_{3}$ and $V^{\prime}\left(\tilde{x}_{1}\right)>0, V^{\prime}\left(\tilde{x}_{2}\right)<0$, and $V^{\prime}\left(\tilde{x}_{3}\right)>0$. Thus, the equilibria corresponding to $\tilde{x}_{1}$ and $\tilde{x}_{3}$ are locally stable, while the equilibrium corresponding to $\tilde{x}_{2}$ is unstable.

\section{Symmetric Case: The Highest Threshold for $\mu$}

In Figure 6 in Section D we see that for $\alpha \in(0,1)$ if $\mu \geq 1$ then the pattern of labor allocations is a pitchfork. Here we show why the threshold for this pattern is necessarily 1 (but we do not formally establish this pattern).

In the symmetric case function $V(x)$ is given by

$$
V(x)=(1-\alpha) \ln x+\mu \ln \left(\frac{1+\phi x}{\phi+x}\right)+\alpha \ln \left(\frac{1+d x}{d+x}\right),
$$

and its derivative is given by

$$
V^{\prime}(x)=\frac{1-\alpha}{x}-\frac{\left(1-\phi^{2}\right) \mu}{(1+\phi x)(\phi+x)}-\frac{\left(1-d^{2}\right) \alpha}{(1+d x)(d+x)} .
$$

In the symmetric case, $x=1$ is always a solution to $V(x)=0$, and there can be two or four more solutions. For each $x$ that solves $V(x)=0$ we also have that $x^{-1}$ solves $V(x)=0$. Since $\alpha \in(0,1)$ we have $\lim _{x \rightarrow 0} V(x)=-\infty$. This implies that $V^{\prime}(1)<0$ if and only if there exists exactly one $\tilde{x}<1$ solving $V(x)=0$. This means that there are exactly three solutions to $V(x)=0$ if and only if $V^{\prime}(1)<0$.

We have $V^{\prime}(1)<0$ if and only if

$$
\mu>\frac{1+\phi}{1-\phi} \cdot \frac{1-2 \alpha+d}{1+d} .
$$

The limit as $\phi \rightarrow 0$ of the right-hand side of the above inequality is 1 . Thus, if $\mu>1$ then for all large enough trade costs the above inequality holds. We see in Figure 6 that 
for large enough trade costs ( $\phi$ close to 0 ), we have a unique equilibrium for $\mu$ smaller some threshold and three equilibria for $\mu$ larger than this threshold. The argument above implies that the only possibility for the value of this threshold is 1. 